\title{
Changes in Paired-Pulse Facilitation Suggest Presynaptic Involvement in Long-Term Potentiation
}

\author{
Paul E. Schulz, ${ }^{1,2}$ Erik P. Cook, ${ }^{2}$ and Daniel Johnston ${ }^{2}$ \\ 'Department of Neurology and the ${ }^{2}$ Division of Neuroscience, Baylor College of Medicine, Houston, Texas 77030
}

Long-term potentiation (LTP) is a use-dependent form of synaptic plasticity that is of great interest as a potential cellular substrate underlying memory. It is important to determine the pre- and/or postsynaptic locus of LTP expression in order to study its underlying mechanisms. Despite intensive investigation, however, its locus of expression remains uncertain. It has been hypothesized that if LTP expression includes a presynaptic locus then it may alter the expression of another presynaptically mediated form of potentiation like paired-pulse facilitation (PPF), which is an increase in a second population excitatory postsynaptic potential when it is elicited shortly after a first. Previous authors have found no change in PPF in association with LTP. We re-examined the hypothesis, however, to reconcile the negative PPF data with other data that have suggested presynaptic involvement in LTP. Extracellular recordings were made in area CA1 of the rat hippocampal slice preparation.

Surprisingly, PPF both increased and decreased significantly in association with LTP. The changes in PPF occurred in a predictable way, however. They correlated inversely with initial PPF magnitude so that a larger Initial PPF was associated with a decrease in PPF with LTP while a smaller initial PPF was associated with an increase. Because PPF increased or decreased in individual slices in association with LTP, the average PPF of all slices did not change, in agreement with previous studies. The changes in PPF were also specific to LTP; that is, they were input specific, were not due to changes in inhibition or nonspecific effects of highfrequency stimulation, were not due to active postsynaptic currents or their nonlinear summation, and PPF changed with the same time course as LTP.

We conclude that the mechanism of early LTP expression includes at least the presynaptic locus. Two hypotheses regarding the presynaptic mechanism underlying LTP expression, which are consistent with finding both increases and decreases in PPF with LTP, are (1) that there is an increase in the number of release sites with LTP or (2) that there is an increase in both the number of release sites and the probability of neurotransmitter release. Increases in the probability of neurotransmitter release alone would not ap-

\footnotetext{
Received Oct. 29, 1993; revised Feb. 15, 1994; accepted Feb. 24, 1994.

We thank Glen Blaschke for tcchnical assistance, Mahmud Haque for computer assistance, Stephen Williams and Costa Colbert for review of the manuscript, and Russell Fricke for the golgi-stained neuron used for modeling. This work was supported by NIH Grants AG00432, AG08664, MH44754, MH48431, and NS11535.

Correspondence should be addressed to Paul E. Schulz, M.D., Department of Neurology, NB-302, One Baylor Plaza, Houston, TX 77030.

Copyright (C) 1994 Society for Neuroscience $0270-6474 / 94 / 145325-13 \$ 05.00 / 0$
}

pear to account for our findings since such increases have been associated only with decreases in PPF. Our findings do not exclude additional postsynaptic involvement. Because of the simplicity of the PPF technique, the few assumptions involved in using it, and the apparent validity of those assumptions, this would appear to be some of the strongest evidence yet for presynaptic involvement in LTP.

[Key words: long-term potentiation (LTP), paired-pulse facilitation (PPF), presynaptic, rat, hippocampus, CA1, synaptic plasticity, memory]

Long-term potentiation (LTP) is a use-dependent form of synaptic plasticity that has been the subject of much recent study as a potential cellular substrate underlying memory formation (Teyler and DiScenna, 1984, 1987; Brown et al., 1988; Madison et al., 1991). To better study its underlying mechanisms, it is important to establish the site of change responsible for its expression. The locus of LTP expression is thought to reside at the synapse since its expression is not associated with changes in the electrophysiological parameters of pre- or postsynaptic neurons (Andersen et al., 1980); however, conflicting evidence has been obtained thus far regarding involvement of the preand/or postsynaptic locus in LTP cxpression.

Presynaptic involvement in LTP expression has been suggested by the observations that LTP is associated with increased transmitter release measured biochemically (Skrede and MaltheSorenssen, 1981; Dolphin et al., 1982), an increase in the probability of transmitter release determined by quantal analysis (Bekkers and Stevens, 1990; Malinow and Tsien, 1990), an increase in the frequency of miniature synaptic currents in hippocampal cultures with glutamate-induced potentiation (Malgaroli and Tsien, 1992), concomitant changes in NMDA and non-NMDA current amplitudes (Bashir et al., 1991), phosphorylation of a presynaptic protein (Routtenberg et al., 1985; Nelson et al., 1989), and involvement of both a presynaptic G-protein (Goh and Pennefather, 1989) and a persistently active kinase (Malinow et al., 1989).

Several experiments using similar techniques have produced opposite results, however, thereby supporting postsynaptic involvement in LTP expression. These results have included finding that LTP is associated with no increase in transmitter release (Aniksztejn et al., 1989), differential changes in NMDA and non-NMDA currents (Kauer et al., 1988; Muller and Lynch, 1988), no change in paired-pulse facilitation (PPF) (McNaughton, 1982; Anwyl et al., 1989; Muller and Lynch, 1989; Zalutsky and Nicoll, 1990; Ghijsen and Da Silva, 1991; Manabe et al., 1993; and see below), and changes in quantal amplitude (Foster and McNaughton, 1991), miniature postsynaptic current amplitudes (Manabe et al., 1992), glutamate receptor numbers 
Table 1. Summary of solutions

\begin{tabular}{llll} 
Solution & $\mathrm{CaCl}_{2}(\mathrm{mM})$ & $\mathrm{MgCl}_{2}(\mathrm{mM})$ & $\begin{array}{l}\text { Picrotoxin } \\
(10 \mu \mathrm{M})\end{array}$ \\
\hline Normal-calcium & 1.5 & 1.2 & No \\
Moderate-calcium & 3.0 & 3.0 & Yes \\
High-calcium & 4.5 & 1.5 & Yes \\
High-magnesium & 1.5 & 4.5 & Yes
\end{tabular}

The operational names of the four solutions used in the subsequent experiments are shown in the left column. The solutions are identical except for their calcium chloride and magnesium chloride concentrations, as noted, and the presence or absence of picrotoxin, which decreases $\mathrm{GABA}_{A}$-mediated inhibition (see Materials and Methods for the other solution consitituents).

(Baudry et al., 1980), and postsynaptic dendritic spine shape (Harris et al., 1989). Additional evidence points to involvement of pre- and postsynaptic elements in LTP expression (Kullmann and Nicoll, 1992), possibly with differing time courses (Davies et al., 1989) or differing probabilities of involvement (Larkman et al., 1992; Liao et al., 1992).

Despite intensive investigation, then, the pre- and/or postsynaptic locus underlying LTP expression remains uncertain. One explanation for the conflicting results may be that many of the techniques used are difficult to perform or interpret and involve assumptions that are difficult to evaluate. Thus, it would be advantageous to use a simple tcchnique with few assumptions, most of which can be tested, to study the locus of LTP expression. Many of these desirable features may be met by using PPF to study the locus of LTP expression.

It has been hypothesized that if the LTP expression mechanism includes a presynaptic locus, then it might alter the expression of another presynaptic form of potentiation. PPF is a short-lasting $(<1 \mathrm{sec})$ increase in a second evoked population excitatory postsynaptic potential (pEPSP) when it follows shortly after a first, and is thought to have a presynaptic locus in area CAl of the hippocampus (Foster and McNaughton, 1991; Storm, 1992). It has been hypothesized that if LTP includes the presynaptic site, then it might be associated with decreases in PPF because other maneuvers that increase the probability of transmitter release are associated with decreases in PPF. Such maneuvers have included protein kinase $\mathrm{C}$ activation by phorbol esters (Asztely et al., 1990), increascd extraccllular calcium (Asztely et al., 1990), and post-tetanic potentiation (PTP) (Anwyl et al., 1989), which is a decremental form of potentiation with a duration of seconds to minutes that follows a train of stimuli and is thought to result from a sustained elevation of presynaptic calcium (Augustine et al., 1987; Delaney et al., 1989; Swandulla et al., 1991).

Average PPF has not been reported to decrease, however, in association with NMDA receptor-dependent LTP (McNaughton, 1982; Anwyl et al., 1989; Muller and Lynch, 1989; Zalutsky and Nicoll, 1990; Ghijsen and Da Silva, 1991; Manabe et al., 1993), with one exception (Voronin and Kuhnt, 1990). While previous studies have failed to demonstrate a change in PPF in association with LTP, the question deserves further examination because (1) it is important to establish the pre- or postsynaptic locus of LTP expression to further study its underlying mechanisms, (2) the lack of change in PPF has been used to support the hypothesis of postsynaptic involvement in LTP, (3) the reason for the conflict between a number of studies that have suggested a change in presynaptic parameters with LTP (Bekkers and Stevens, 1990; Malinow and Tsien, 1990) and the negative findings regarding changes in PPF needs to be resolved, (4) PPF has been reported to change in association with LTP at other synapses (Krug et al., 1989; Staubli et al., 1990; Christie and Abraham, 1994) so that a lack of change in CA1 would suggest that there is something fundamentally different about CAI LTP, and (5) the method of using PPF is simple and has few assumptions so that a change in PPF would provide strong evidence for presynaptic involvement in LTP. Thus, we retested the hypothesis that LTP expression alters PPF and found that PPF was altered in association with LTP, suggesting that the locus of LTP expression includes at least a presynaptic component.

\section{Materials and Methods}

Preparation of hippocampal slices. Brains from adult Sprague-Dawley rats $(150-200 \mathrm{gm})$ were quickly removed and placed in iced saline. The hippocampi were dissected out and $375-\mu$ m-thick slices were made perpendicular to the septotemporal axis with a Mcllwain tissue chopper. Slices were transferred to a Haas-type (Haas et al., 1979) interface recording chamber (Medical Systems) and maintained using standard procedures (Williams and Johnston, 1990).

The effect of altering bath calcium and magnesium on a number of parameters was tested. For simplicity, the salines will be referred to as normal-, moderate-, and high-calcium, and high-magnesium. The normal-calcium solution contained (in $\mathrm{mM}$ ) $\mathrm{NaCl}, 120 ; \mathrm{KCl}, 3 ; \mathrm{NaHCO}_{3}$, 23; dextrose, $11 ; \mathrm{CaCl}_{2}, 1.5$; and $\mathrm{MgCl}_{2}, 1.2$. The other three solutions were the same, except that $10 \mu \mathrm{M}$ picrotoxin was added to the solutions to block $\mathrm{GABA}_{\mathrm{A}}$-mediated inhibition, and the $\mathrm{CaCl}$, and $\mathrm{MgCl}_{2}$ concentrations were adjusted as follows (see Table 1): for the moderatecalcium solution, $\mathrm{CaCl}_{2}$ and $\mathrm{MgCl}_{2}$ were $3 \mathrm{~mm}$; for the high-calcium solution, $\mathrm{CaCl}_{2}$ was $4.5 \mathrm{~mm}$ and $\mathrm{MgCl}_{2}$ was $1.5 \mathrm{~mm}$; and for the highmagnesium solution, $\mathrm{CaCl}_{2}$ was $1.5 \mathrm{~mm}$ and $\mathrm{MgCl}_{2}$ was $4.5 \mathrm{~mm}$. As indicated, the bathing solution sometimes contained $200 \mu \mathrm{M}$ 2-hydroxysaclofen (Research Biochemicals, Natick, MA) to reduce GA$\mathrm{BA}_{\mathrm{B}}$-mediated inhibition, $100 \mu \mathrm{M}$ D,L-aminophosphonovalerate (APV; Research Biochemicals) to block NMDA receptors, or 1-10 $\mu_{\mathrm{M}}$ 6-cyano7-nitroquinoxaline-2,3-dione (CNQX; Research Biochemicals) to reduce $\alpha$-amino-3-hydroxy-5-methyl-4-isoxazole propionic acid (AMPA) currents. CNQX (1-10 mM) was dissolved initially as a 10\% dimethyl sulfoxide (DMSO) stock solution, which was diluted 100-1000-fold for experimental use (final DMSO concentrations were $0.1-0.01 \%$ ). The solutions were gassed with $95 \% \mathrm{O}_{2}, 5 \% \mathrm{CO}_{2}$. The temperature of the bathing solution was $\sim 33^{\circ} \mathrm{C}$.

Extracellular recordings. Microelectrodes were pulled from $1.5 \mathrm{~mm}$ o.d. glass tubing using a Narishige puller and were filled with $750 \mathrm{~mm}$ $\mathrm{NaCl}$ (resistances were 1-5 M 2 ). Stimulation was given at 15-30 sec intervals via bipolar, Teflon-coated platinum stimulating electrodes (World Precision Instruments stimulus generator and isolator; stimulus duration $=50 \mu \mathrm{sec}$ ). Extracellular pEPSP recordings were made from stratum radiatum in area $\mathrm{CAl}$ and the data were filtered at $5 \mathrm{kHz}$ and recorded on line with a DEC-11/23 computer for later analysis using software written in Basic-23.

Stable pEPSPs were obtained with a stimulus intensity that initially yielded a $1.3 \mathrm{mV}$ pEPSP, though for specific experiments the range varied from 0.9 to $2.6 \mathrm{mV}$ as indicated. The pEPSPs were controlled by selecting the initial pEPSP amplitude rather than percentage of maximal stimulation needed to induce the pEPSPs; however, the $2.6 \mathrm{mV}$ pEPSPs were obtained with about half-maximal stimulation. In each experiment, a single stimulus intensity was followed and used for highfrequency stimuli (HFS) in an effort to stimulate the same axons throughout the experiment.

PPF was initially measured across a range of interstimulus intervals (ISIs; two to six traces for each ISI, ISIs of 15-400 msec). HFS was then given until LTP was saturated, and PPF was remeasured $20 \mathrm{~min}$ after the last HFS with the same ISIs. To address the time course of change in PPF with LTP, PPF was measured with a single ISI $(55 \mathrm{msec})$ at 20 sec intervals throughout the experiment. The results of both groups were combined as indicated.

HFS consisted of 10 trains $(100 \mathrm{~Hz}, 50 \mathrm{msec})$ delivered at $200 \mathrm{msec}$ intervals for a total of 50 stimulations over $2 \mathrm{sec}$. To achieve maximal LTP, two paradigms were used. Initially HFS was given at $20 \mathrm{~min}$ 
intervals until no additional LTP was elicited. We were concerned that advanced slice age might affect PPF, because LTP saturation could require $1-3 \mathrm{hr}$ to achieve with this paradigm. Consequently, additional slices were given six HFS at 1 min intervals, followed by HFS at 20 min intervals until no additional LTP was elicited. In these experiments, LTP saturation was typically achieved by the initial six HFS, which was verified by the lack of additional LTP with subsequent HFS. The results of using both paradigms were combined as indicated.

Data analysis. To obtain suitable pEPSPs, an attempt was made to identify clearly the fiber volley as being separate from the initial pEPSP slope. Any experiments in which a change occurred in fiber volley or stimulus artifact were excluded from analysis. pEPSP traces were analyzed by obtaining the maximum initial pEPSP slope occurring after the fiber volley. The maximum slope was obtained by linear regression over the points obtained over a range of $0.4-0.6 \mathrm{msec}$ on the initial slope. To avoid bias, the same time points on the initial slope of the pEPSP were analyzed throughout the experiment. PPF was calculated as the slope of the second pEPSP minus the first, divided by the first. $T$ tests were used to compare the PPF elicited under different conditions with one point having been taken from each experiment before and after a manipulation. A linear regression test was used for examining correlations.

Modeling. For the neural modeling seen in Figure 8, the neural simulation program NEURON (Hines, 1989) was used to simulate a 149 compartment CA3 pyramidal neuron reconstructed from a Golgi-stained neuron (Spruston et al., 1993). The parameters used in this fully passive model were $R_{m}=60,000 \Omega \mathrm{cm}^{2}, C_{m}=1 \mu \mathrm{F} / \mathrm{cm}^{2}, R_{i}=200 \Omega \mathrm{cm}$, and the resting membrane potential $=-65 \mathrm{mV}$. Fifty synapses were placed uniformly across the apical dendritic tree. The synapses were each modeled as single, simultaneous conductance changes using an $\alpha$ function $(1 / \alpha=2 \mathrm{msec})$ and a synaptic reversal potential of $0 \mathrm{mV}$. Separate simulations were run for individual synaptic conductances that ranged from $10 \mathrm{pS}$ to $2 \mathrm{nS}$, which resulted in total peak synaptic conductances ranging from $0.5 \mathrm{nS}$ to $100 \mathrm{nS}$. It is estimated that a typical single physiological synaptic conductance change is on the order of $100 \mathrm{pS}$, so the maximum of $2 \mathrm{nS} /$ synapse assumed in this model is probably well above the physiologic range. For each simulation, average peak and slope measurements for both synaptic potential and synaptic current were calculated from 10 randomly selected synapses. Slope measurements were derived from the first $0.5 \mathrm{msec}$ of each synaptic event.

\section{Results}

$P P F$ changed in a predictable way with LTP

PPF changed in association with LTP. Surprisingly, it both increased and decreased. The change in 63 slices is demonstrated in Figure $1 A$. PPF, with an ISI of $55 \mathrm{msec}$, was measured at baseline and after LTP was induced by one or multiple HFS. Multiple HFS were administered to some slices to induce LTP saturation. Initial PPF is plotted on the $\mathrm{x}$-axis and the change in PPF associated with LTP is plotted on the y-axis. The change in PPF was calculated as final PPF minus initial PPF; thus, initial and final PPF values of 50 and 30, for example, are plotted as $50,-20$. Rather than randomly increasing or decreasing, the changes in PPF with LTP exhibited an inverse relationship with initial PPF as demonstrated by the best-fit linear regression line plotted in dashes (Fig. 1A; $r^{2}=0.63, p<0.0005, n=63, y=$ $-0.70 x+17.9, \mathrm{x}$-intercept $=25.6$ ). Thus, larger initial PPF was associated with a decrease in PPF with LTP while smaller initial PPF was associated with an increase in PPF. If there were no changes in PPF with LTP, the linear regression line would have been horizontal.

\section{Similar amounts of LTP were associated with similar changes in $P P F$}

The finding that PPF could increase, decrease, or remain the same in association with LTP was surprising and raised the possibility that the changes in PPF were unrelated to LTP. We had excluded any slices showing a change in fiber volley or stimulus artifact so that the observed changes in PPF would not
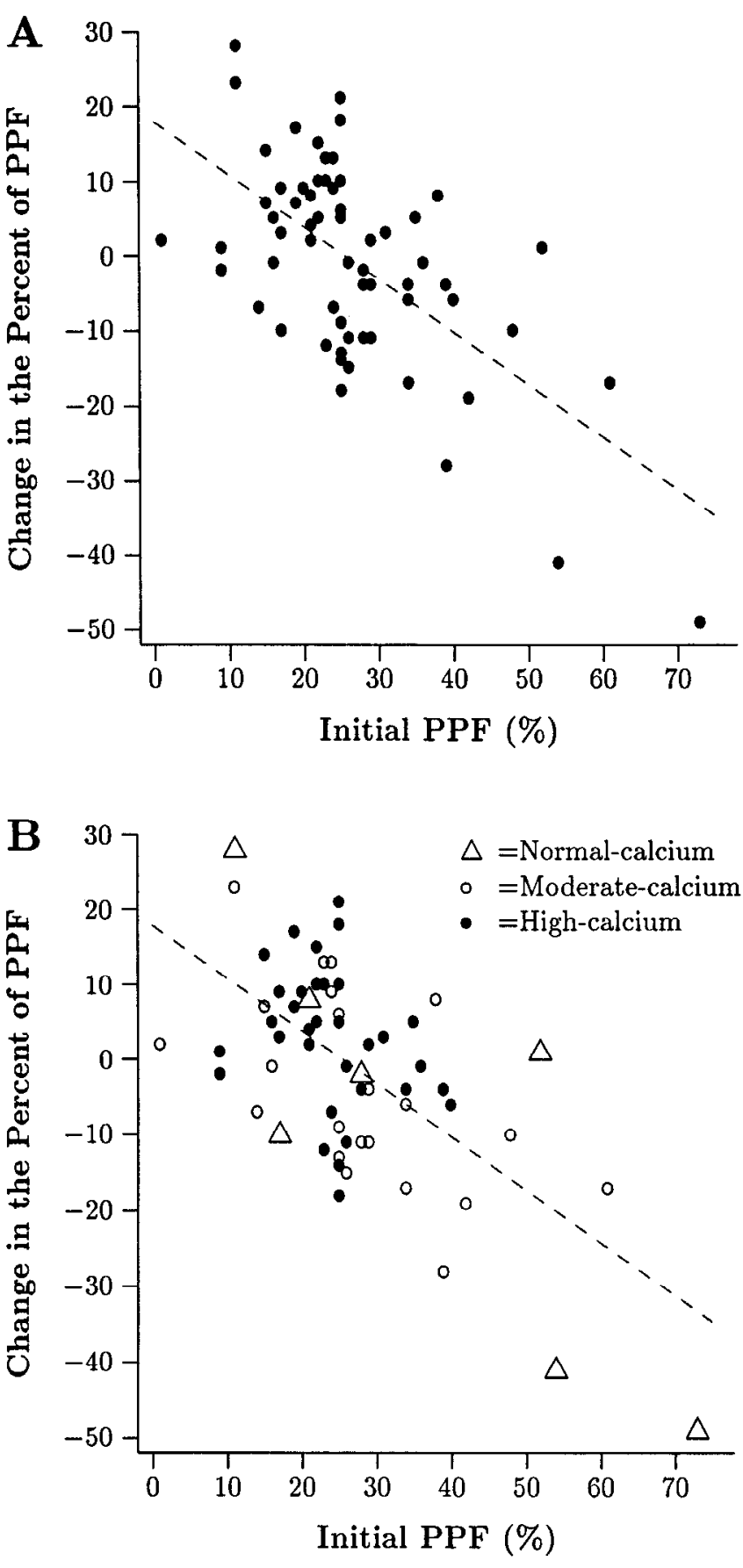

Figure 1. Paired-pulse facilitation (PPF) changed in association with long-term potentiation (LTP). $A$, PPF elicited with a $55 \mathrm{msec}$ interstimulus interval (ISI) was measured at baseline and after LTP induction, which was achieved by administering one or several high-frequency stimuli (HFS). The initial PPF is plotted on the $x$-axis and the change in PPF is plotted on the y-axis (change = final PPF minus initial PPF; thus, e.g., a change in PPF from $50 \%$ to $30 \%$ is plotted as $50,-20$ ). PPF changed in association with LTP, and there was a significant inverse correlation betwecn initial PPF and the change in PPF with LTP $(n=$ $63, r^{2}=0.63, p<0.0005$ ). The best-fit linear regression line is plotted (dashed line; $y=-0.70 x+17.9$ ). If there had been no relationship between PPF and LTP, the regression line would have been horizontal. $B$, The data from $A$ were obtained in three solutions, which are identified in $B$. While the range of initial PPF values differed by solution, being narrowest in high-calcium, similar changes in PPF were associated with LTP in all solutions. 

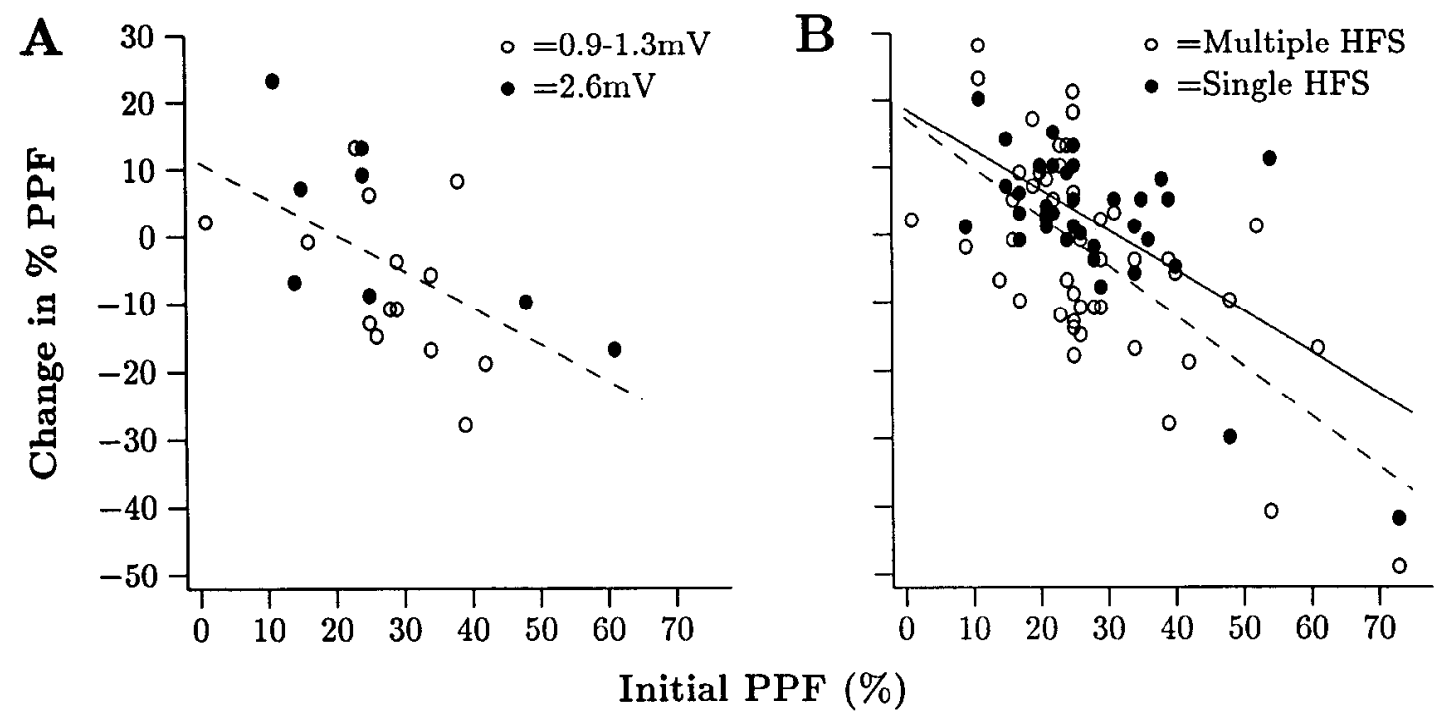

Figure 2. The change in PPF versus the magnitude of LTP induced. $A$, The same magnitude of LTP was induced in slices given HFS with a low stimulus intensity (0.9-1.3 mV pEPSPs) and a high intensity (2.6 mV pEPSPs), and both groups had the same changes in PPF. The experiments were performed in the moderate-calcium solution. The best-fit linear regression line for all points was $y=-0.54 x+11.0$ and is shown in dashes for ease of comparison of the groups $\left(r^{2}=0.55, n=22, p<0.005\right)$. B. More LTP was induced by multiple HFS versus a single HFS, and a greater change in PPF accompanied the multiple HFS as demonstrated by the greater slope of the best-fit linear regression line for multiple HFS (O, dashed line; slope $\left.=-0.73, n-50, r^{2}=0.66, y=-0.73 x+17.4, p<0.0005\right)$ versus single HFS (0, solid line; slope $=-0.60, n=35, r^{2}=0.66, y=$ $-0.60 x+18.7, p<0.0005 ; 22$ of the experiments were in both groups).

have been due to processes like nonspecific short-term changes in excitability or movement of the slices. To be morc ccrtain of the specificity of the relationship between the changes in PPF and LTP, however, we went on to perform multiple tests of the specificity of changes in PPF to LTP.

A first test of specificity was to examine whether similar amounts of LTP were associated with similar changes in PPF, and conversely whether different amounts of LTP were associated with different changes in PPF. Similar amounts of LTP were induced by HFS in the normal-, moderate-, and highcalcium solutions (P. E. Schulz and D. Johnston, unpublished observations). The changes in PPF observed in the three solutions are combined in Figure $1 A$ and separated in Figure $1 B$. The high-calcium solution had a narrower range of initial PPF values than was observed in the other solutions; nonetheless, PPF increased and decreased similarly in association with LTP in all solutions with only the initial PPF value appearing to determine the change in PPF associated with LTP.

Similar amounts of LTP were also induced by HFS at lower (0.9-1.3 mV pEPSPs) versus higher (2.6 mV pEPSPs) stimulus intensity in moderate-calcium (Schulz and Johnston, unpublished observations), and Figure $2 A$ demonstrates that the associated changes in PPF in the two groups were also similar. A regression line for all points is drawn for convenient comparison $\left(y=-0.54 x+11.0, r^{2}=0.55, n=22, p<0.005\right)$.

More LTP was induced by multiple HFS than by single HFS, and its effect on changes in PPF is examined in Figure $2 B$. There were greater changes in PPF associated with multiple HFS $(O$, $n=50, y=-0.73 x+17.38$, dashed line, $r^{2}=0.66, p<0.0005$ ) than single HFS $\left(\odot, n=35, y=-0.60 x+18.7\right.$, solid line, $r^{2}$ $=0.66, p<0.0005)$, as indicated by the steeper slope of its regression line $(-0.73$ for multiple HFS vs -0.60 for single HFS). In 22 slices, PPF was measured under both circumstances so that the total number of points is greater than in Figure 1. Thus, similar amounts of LTP were associated with similar changes in PPF (Figs. $1 B, 2 A$ ) and greater amounts of LTP were associated with greater changes in PPF (Fig. $2 B$ ).

\section{PPF changed with the same time course as LTP}

A second test of the specificity of the changes in PPF to LTP was to examine whether the changes in PPF had a similar time course to the onset of LTP; that is, if the changes in PPF were specific to LTP, then PPF and LTP should change with the same time course. The time courses of both increases and decreases in PPF associated with LTP were examined.

The time course of increases in PPF associated with LTP are shown in Figure 3. As LTP was saturated in the high-calcium solution, PPF was examined with a single ISI ( $55 \mathrm{msec})$ throughout the experiments. The initial pEPSP magnitudes were 1.3 $\mathrm{mV}$. PPF was measured at $20 \mathrm{sec}$ intervals throughout the experiments in Figure 3 and was averaged and plotted in $5 \mathrm{~min}$ bins, except for the first point after each HFS, which contained PTP and is the average of a single trace from each experiment. One experiment in which PPF increased in association with LTP is shown in Figure 3, $A$ and $B$. Eight HFS (arrows) increased the pEPSP slopes by $79 \%$ (Fig. $3 A$ ) and were associated with an increase in PPF from $15 \pm 2 \%$ to $25 \pm 1 \%$ that persisted for 3 hr after the last HFS (Fig. 3B).

Nonlinear summation of postsynaptic potentials would not account for increases in PPF; however, to confirm that other active postsynaptic currents were not responsible for the changes in PPF associated with LTP, pEPSP slopes were returned to baseline after LTP saturation by wash-in of $1 \mu \mathrm{M}$ of the AMPA receptor antagonist CNQX. Despite returning the pEPSP slopes to baseline (Fig. $3 A$ ), PPF remained elevated for the duration of this experiment (Fig. $3 B$ ). Representative voltage traces from this experiment are shown in Figure $3 C$. Each is an average of several traces obtained at the times indicated in Figure $3 A$ by small letters, that is, at baseline (a), and after LTP saturation and return of the pEPSP to almost the original size with CNQX 
A

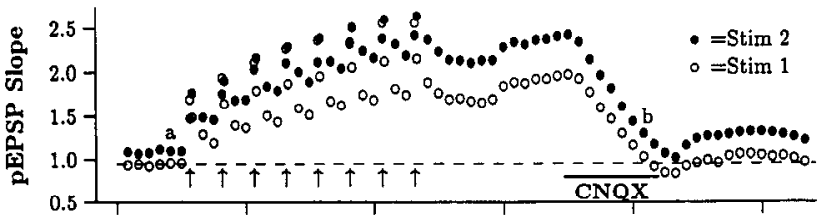

B

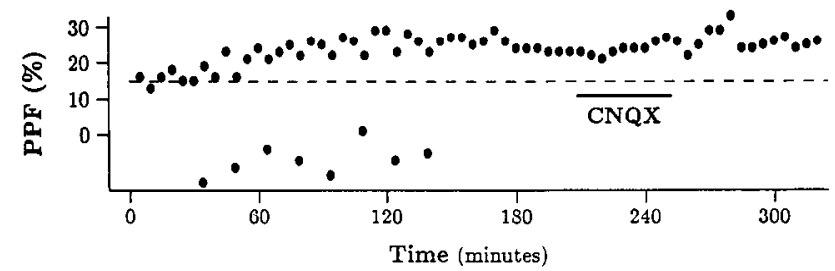

C

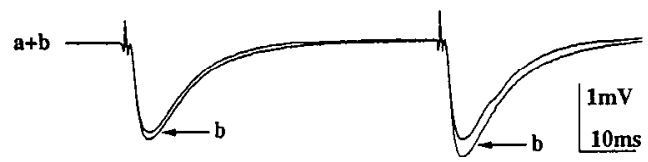

D

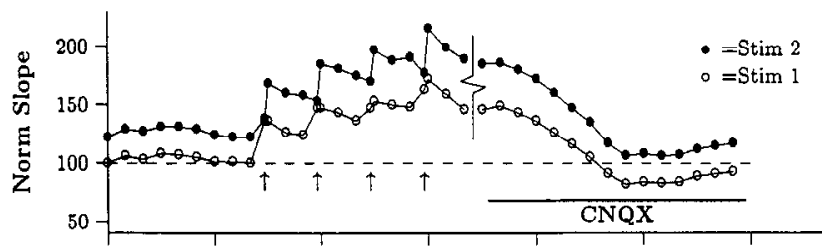

$\mathbf{E}$

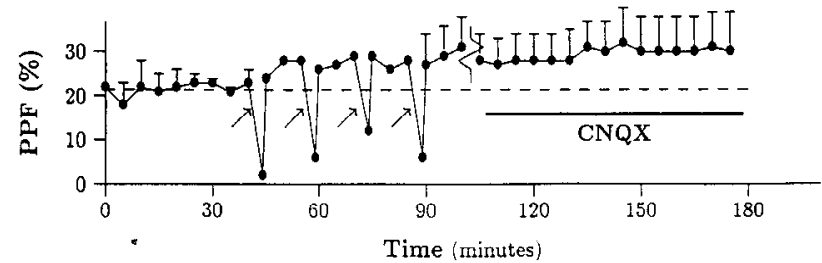

Figure 3. Increases in PPF occurred shortly after HFS and persisted. $A$, The pEPSP slopes (mV/msec) elicited by the first (Stim I) and second (Stim 2) paired-pulse stimuli are plotted over time for a single experiment. Data were sampled at $20 \mathrm{sec}$ intervals throughout each experiment in this and the subsequent figures, and each point plotted is the average of 5 min of data, except for the first point after each HFS, which contains PTP and is the average of a single trace from each experiment. Eight HFS (arrows) administered at 15 min intervals in high-calcium resulted in 74\% LTP. After LTP was saturated, $1 \mu \mathrm{M}$ 6-cyano-7-nitroquinoxaline-2,3-dione $(C N Q X)$ was washed in to return the pEPSP slopes to baseline. $B$, PPF increased in association with LTP induction from 14.6 $\pm 2.2 \%$ at baseline to $25.2 \pm 0.5 \%$ after the eighth HFS (ISI $=55 \mathrm{msec}$ ). PPF remained elevated for the $3 \mathrm{hr}$ duration of the experiment after the last HFS, including when the pFPSP slopes were returned to baseline by CNQX. $C$, Representative raw voltage traces from the experiment above, obtained at the times indicated in small letters, are shown. The superimposed traces demonstrate that PPF increased in association with LTP. $D$, HFS (arrows) at $15 \mathrm{~min}$ intervals increased the normalized pEPSP slopes for eight slices by $46 \%$ (points are mean \pm SEM). After LTP was saturated, $1-3 \mu \mathrm{M}$ CNQX returned the initial pEPSP slopes to their baseline. $E$, PPF of the slices in $D$ increased with LTP from $21.8 \pm 1.6 \%$ to $30.9 \pm 2.5 \%$ (ISI $=55 \mathrm{msec}, n=8, p=0.01$ by $t$ test). Wash-in of CNQX after LTP saturation depressed synaptic transmission by $43 \%$, but did not alter PPF (average $=30.5 \pm 2.9 \%, p=0.89$ by $t$ test), which was still different from baseline ( $p=0.02$ by $t$ test). This suggests that neither the induction of postsynaptic currents nor their nonlinear summation can account for the changes in PPF associated with LTP.
A

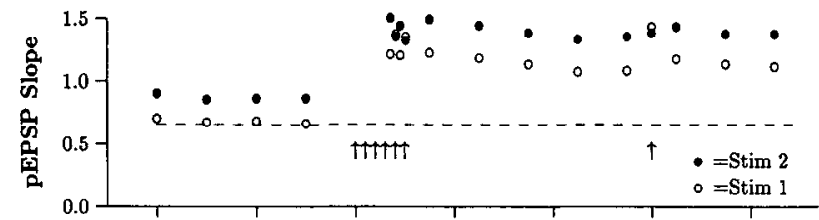

B

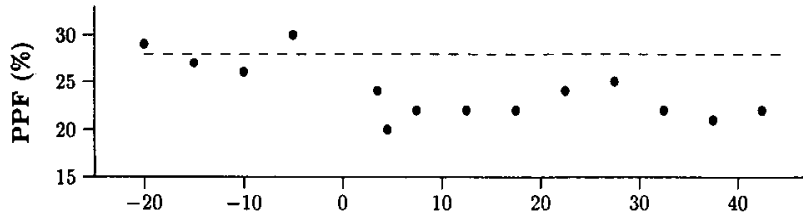

C

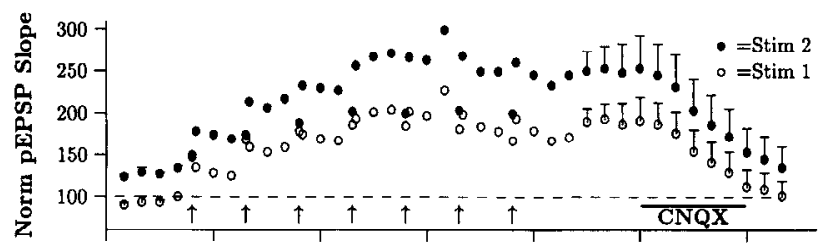

D

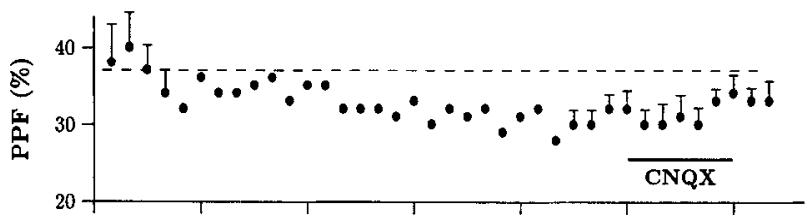

$\mathbf{E}$

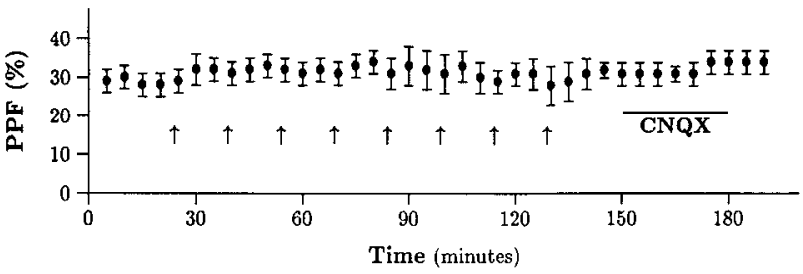

Figure 4. Decreases in PPF occurred shortly after HFS and persisted. $A$, Six HFS were administered to one slice over $6 \mathrm{~min}$ (arrows) and this was associated with a $64 \%$ increase in the $\mathrm{pEPSP}$ slope (in $\mathrm{mV} / \mathrm{msec}$, moderate-calcium solution). A seventh HFS was administered $25 \mathrm{~min}$ later to be certain that LTP was saturated. $B$. PPF in the slice in $A$ decreased shortly after HFS from $28 \pm 0.9 \%$ at baseline to $22.4 \pm 0.5 \%$ after LTP induction and remained depressed for the duration of the experiment. Each point is the average of $5 \mathrm{~min}$ of data, except for the points between HFS 4 and 6 , which are individual traces. $C$, Ninety percent LTP was induced in the four slices given HFS in high-calcium that showed a decrease in PPF. Wash-in of CNQX after LTP saturation returned pEPSP slopes to baseline. $D$, LTP was associated with a decrease in PPF from $37 \pm 2 \%$ to $31 \pm 1 \%$ (ISI $=55 \mathrm{msec}$ ). Wash-in of CNQX did not alter PPF. $E$, Averaging PPF from the slices from Figure $3 E$, in which PPF increased with LTP, with the slices from $D$ of this figure, in which PPF decreased with LTP, results in this plot, which would suggest no change in PPF with LTP if the change in individual slices was not examined.

(b). Superimposition of traces a and b in Figure $3 C$ demonstrates that PPF increased in association with LTP. The pEPSP slopes were used for analysis, but on this time scale the change in pEPSP amplitude is more obvious.

Group data for the eight slices in which the time course of PPF was monitored that showed an increase in PPF with LTP are plotted in Figure 3, $D$ and $E$. Initial pEPSP magnitudes were $1.3 \mathrm{mV}$ in all experiments. In these experiments, four HFS (arrows) increased the baseline pEPSP slope by $46 \%$ (Fig. 3D). PPF for the group data increased in association with LTP from 
$\mathbf{A}$

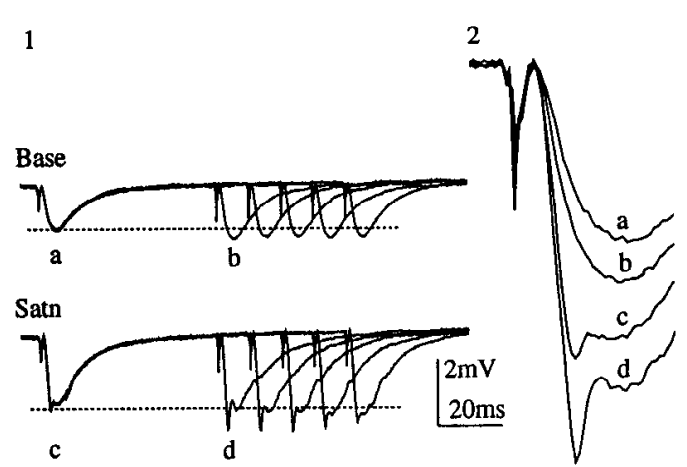

B

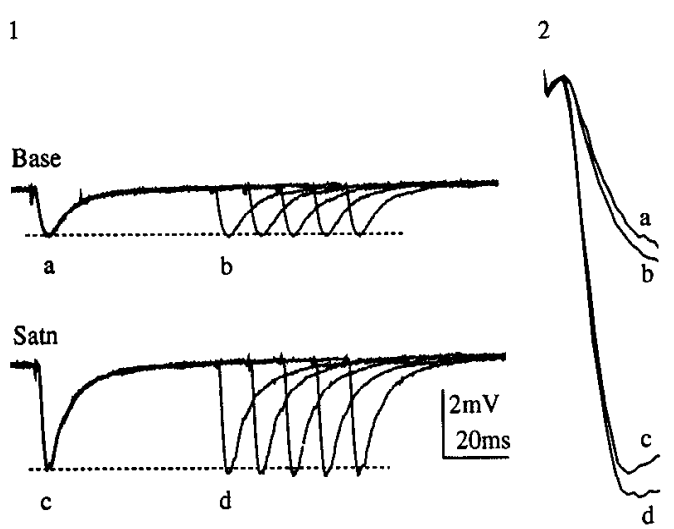

Figure 5. Examples of decreases in PPF associated with LTP. Al, PPF was measured at baseline (Base) with ISIs of $55,65,75,85$, and 95 $\mathrm{msec}$ in a single slice in moderate-calcium. Each plot is an average of two to four traces. The slice was then given HFS until LTP was saturated, and PPF was remeasured over the same ISIs $(S a t n)$. A2, Enlargement and superimposition of the initial slopes of the first and second pairedpulse pEPSPs obtained with the $55 \mathrm{msec}$ ISI demonstrates that PPF decreased after LTP saturation since the increase in slope from $a$ to $b$ is greater than from $c$ to $d$. $B$, PPF also decreased in this second slice in which the experiment was performed in high-calcium.

$21.8 \pm 1.6 \%$ to $30.9 \pm 2.5 \%$ (Fig. $3 E ;$ ISI $=55 \mathrm{msec}, p=0.01$ by $t$ test). Wash-in of CNQX (1-3 $\mu \mathrm{M})$ after LTP saturation again returned pEPSP slope to baseline (Fig. $3 D$; a $43 \%$ decrease), but did not alter PPF (Fig. $3 E$ ). After CNQX wash-in, PPF was $30.5 \pm 2.9 \%$, which was not different from PPF after LTP saturation $(30.9 \pm 2.5 \%, p=0.89$ by $t$ test), but was still different from PPF measured at baseline $(21.8 \pm 1.6 \%, p=0.02$ by $t$ test).

An example of the time course of a decrease in PPF associated with LTP is shown in Figure 4A. LTP was induced with six HFS delivered at $1 \mathrm{~min}$ intervals in the high-calcium solution. The initial pEPSP magnitude was $1.3 \mathrm{mV}$. PPF decreased in association with LTP induction from $28 \pm 1 \%$ at baseline to $22 \pm$ $1 \%$ shortly after the sixth IIFS (Fig. $4 B$; ISI $=55 \mathrm{msec}$ ), and remained depressed for the subsequent $40 \mathrm{~min}$, during which a seventh HFS was given to confirm that LTP was saturated. The points in which PPF was measured during PTP have been removed from Figure 4, $B$ and $D$, for clarity. The four slices in which PPF decreased with LTP and in which the time course was monitored are averaged in Figure 4, $C$ and $D$. HFS (arrows) was given at $15 \mathrm{~min}$ intervals in high-calcium. Ninety percent LTP (Fig. 4C) was associated with a decrease in PPF from 37 $\pm 2 \%$ to $31 \pm 1 \%$ (Fig. $4 D$; ISI $=55 \mathrm{msec}$ ). Wash-in of CNQX after LTP saturation returned pEPSP slopes to baseline (Fig.
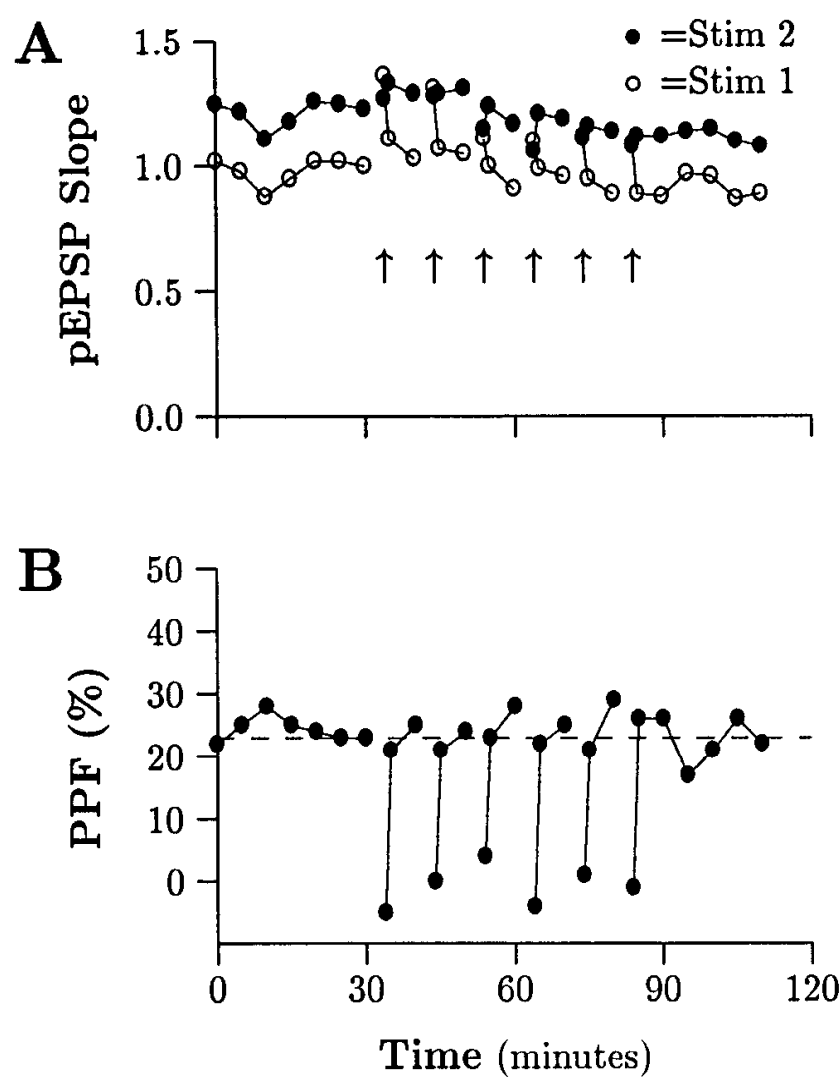

Figure 6. Changes in PPF were not due to nonspecific effects of HFS. $A$, In the presence of D,L-aminophosphonovalerate, six HFS (arrows) at $10 \mathrm{~min}$ intervals did not induce LTP of the first $(O)$ or second $(0)$ pEPSP slopes elicited by the paired-pulse protocol $(n=3$, in $\mathrm{mV} / \mathrm{msec}$, ISI $=55 \mathrm{msec}$ ). $B$, There was also no associated change in PPF.

$4 C$ ) with no obvious change in PPF (Fig. 4D), in agreement with a previous report (Manabe et al., 1993). Examples of raw traces from two experiments in which PPF decreased with LTP are shown in Figure 5. Each trace is an average of two to four traces. In Figure $5 A 1$, PPF was measured at baseline (labeled Base) with ISIs of $55,65,75,85$, and $95 \mathrm{msec}$ in moderatecalcium. The slice was then given HFS until LTP was saturated and PPF was remeasured over the same ISIs (labeled Satn). In Figure $5 A 2$, enlargement and superimposition of the initial slopes of the first and second paired-pulse pEPSPs obtained with the 55 msec ISI demonstrate that PPF decreased after LTP saturation since the increase in slope from $a$ to $b$ is greater than from $\mathrm{c}$ to $\mathrm{d}$. The same was true for a slice examined in highcalcium (Fig. 5B).

The time course of the changes in PPF with LTP, then, was similar to LTP. Despite increases and decreases in PPF, though, there was no change in average PPF associated with LTP. This was demonstrated in two ways. First, the eight slices from Figure $3 E$ in which PPF increased with LTP are averaged with the four slices from Figure $4 D$ in which PPF decreased. The result shown in Figure $4 E$ is that average PPF did not change. The small SEMs would also suggest no change in PPF. Second, average PPF also remained constant over all 63 slices examined and plotted in Figure 1. Initial PPF averaged $26.0 \pm 1.7 \%$ and final PPF averaged $23.8 \pm 1.6 \%$ (mean \pm SEM). These results agree with those of previous authors who found no change in average PPF associated with LTP (McNaughton, 1982; Anwyl et al., 
1989; Muller and Lynch, 1989; Zalutsky and Nicoll, 1990; Ghijsen and Da Silva, 1991; Manabe et al., 1993).

\section{Changes in PPF were not due to nonspecific effects of HFS}

A third test of the specificity of the changes in PPF to LTP was to determine whether the changes in PPF were nonspecifically related to HFS. This was tested in two ways. First, the effect of HFS on PPF after LTP saturation was examined. After LTP saturation, HFS was given to 14 slices from the moderate- and high-calcium groups, and PPF was examined (ISI $=55 \mathrm{msec}$; slices are a subset of those shown in Figure 10). In the absence of eliciting additional LTP, no change in PPF was noted (16 \pm $3 \%$ before HFS vs $17 \pm 4 \%$ at 15 min post-HFS, $n=14$, data not shown).

Second, the effect of HFS on PPF was examined when NMDA receptor-dependent LTP was blocked by APV (Fig. 6). Both HFS paradigms used to induce LTP were tested. In the first, HFS (arrows) was administered six times at 20 min intervals to naive slices in the presence of $100 \mu \mathrm{M}$ D,L-APV. There were no changes in pEPSP slopc during this $2 \mathrm{hr}$ period of repetitive HFS (Fig. 6A), and PPF remained constant at 22\% (Fig. $6 B ; n$ $=3$, ISI $=55 \mathrm{msec}$; each point is the average of $5 \mathrm{~min}$ of data except for the first point after each HFS, which is a single trace containing PTP). The effect of HFS on PPF using the second LTP induction paradigm was tested by giving six HFS at $1 \mathrm{~min}$ intervals to slices bathed in APV, and there was no change in either pEPSP slope or PPF (34-35\%, data not shown, $n=2$ ). Thus, no changes in PPF occurred over a range of initial PPFs (18-40\%) that without APV would have been associated with large changes as in Figure 1. This suggests that the changes we observed in PPF were specific to NMDA receptor-dependent LTP.

\section{Changes in PPF were input specific}

A fourth test of the specificity of changes in PPF to LTP was performed by examining whether the changes in PPF were specific to the input exhibiting LTP. If the changes in PPF were due to an alternate cause, such as calcium precipitation or slice movement, then the changes in PPF would not be specific to the input showing LTP. The input specificity was examined using two stimulating electrodes and one recording electrode in stratum radiatum. The independence of the two inputs was judged by the additivity of pEPSPs and a lack of PPF between the two inputs; however, some overlap could not be excluded. Initial pEPSP magnitudes were about $1.3 \mathrm{mV}$ in both inputs in all slices, and experiments were performed in high-calcium. A single HFS to one input was associated with LTP in that input, and a small decrease in slope in the other input (Fig. 7A;n= 4 pairs). PPF was measured concomitantly in the two inputs and for graphing clarity was normalized by setting the average of the pre-HFS PPF equal to $100 \%$. PPF increased significantly in the input given HFS from $20.5 \pm 2.3 \%$ to $30.0 \pm 2.3 \%$ (Fig. $7 B$, plotted as $100-146 \%$; ISI $=55 \mathrm{msec}, n=4, p=0.04$ ), and did not change in the nonstimulated input $(26.5 \pm 4.5 \%$ to 22.8 $\pm 5.6 \%$, plotted as $100-86 \% ; n=4, p=0.66$ ). This finding indicates that all synapses in the slice did not show the same change in PPF in association with HFS.

\section{Changes in PPF were not due to postsynaptic changes}

A fifth test of the specificity of changes in PPF to LTP was performed by examining whether two possible postsynaptic changes might have altered PPF, that is, activation of postsyn-
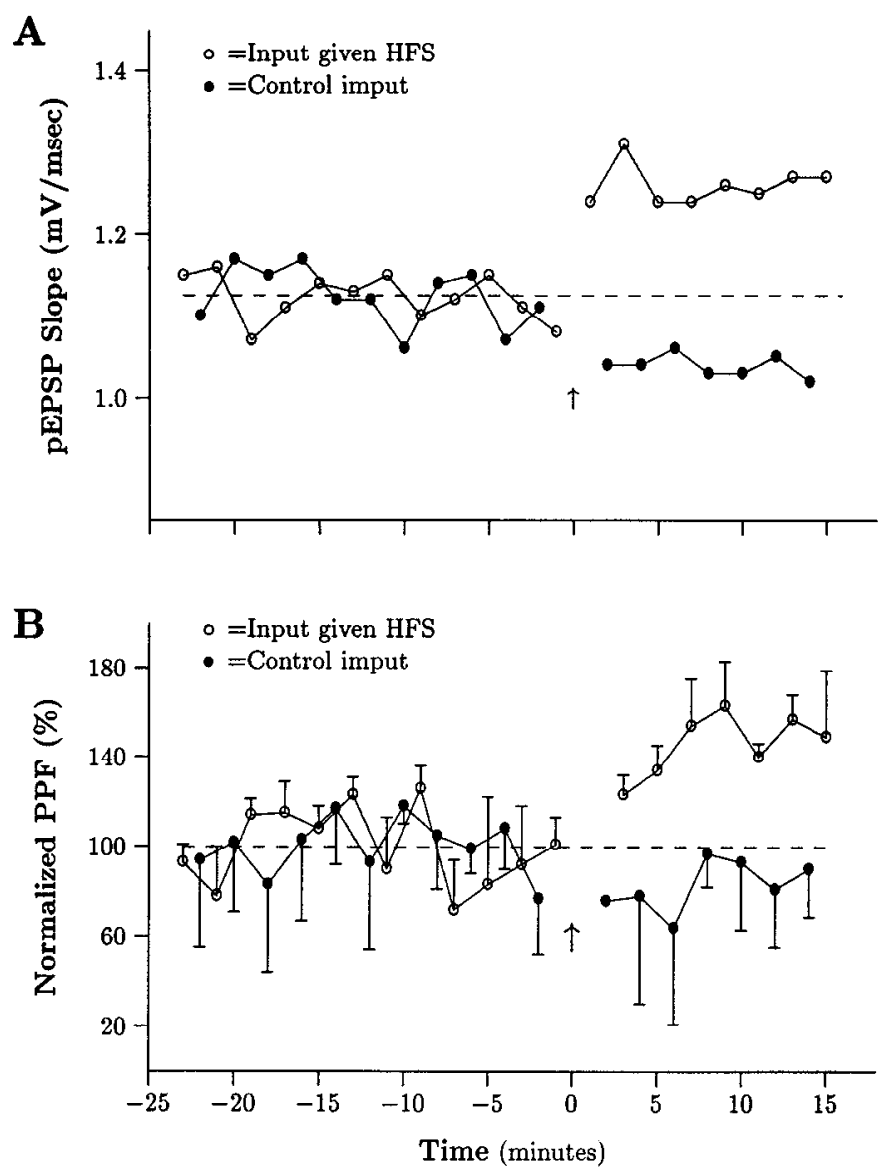

Figure 7. Changes in PPF with LTP were input specific. The pEPSPs elicited by two independent inputs in stratum radiatum were recorded in one electrode. The inputs were independent as judged by the additivity of pEPSPs and a lack of PPF between the two inputs. The possibility of some overlap, however, cannot be ruled out. $A$, Initial pEPSP magnitudes were $\sim 1.3 \mathrm{mV}$ in both inputs in all slices. A single HFS in high-calcium to one input (arrow) was associated with LTP in that input $(O ; n=4)$ and a small decrease in slope in the other input (O). $B$, Normalized PPF (ISI $=55 \mathrm{msec}$ ) increased significantly in the input given HFS from $20.5 \pm 2.3 \%$ to $30.0 \pm 2.3 \%$, a $46 \%$ increase $(n=4$, $p=0.04)$, and did not change in the other input $(26.5 \pm 4.5 \%$ to 22.8 $\pm 5.6 \%, p=0.66, n=4$ ). Thus, LTP and the associated changes in PPF were input specific so that the changes in PPF were not due to a general change in PPF in the slice.

aptic currents or their nonlinear summation. With regard to activation of postsynaptic currents, we showed above that washin of CNQX returned pEPSPs to baseline but did not return PPF to baseline (Figs. 3, 4), suggesting that a change in excitatory postsynaptic currents was not responsible for changes in PPF. The possibility of altered inhibitory currents is addressed in the next section.

Several lines of evidence also argue against nonlinear summation of postsynaptic currents as a cause for the changes in PPF. (1) The nonlinear summation hypothesis predicts that decreasing the $\mathrm{pEPSP}$ to baseline after LTP saturation would return PPF to baseline; however, wash-in of CNQX after LTP saturation did not change whether it had increased (Fig. 3) or decreased (Fig. 4) in association with LTP. (2) Nonlinear summation would explain only decreases in PPF and would not account for the increases observed in PPF. (3) If the observed decreases in PPF were due to nonlinear summation, then other forms of potentiation with a similar initial amplitude should 

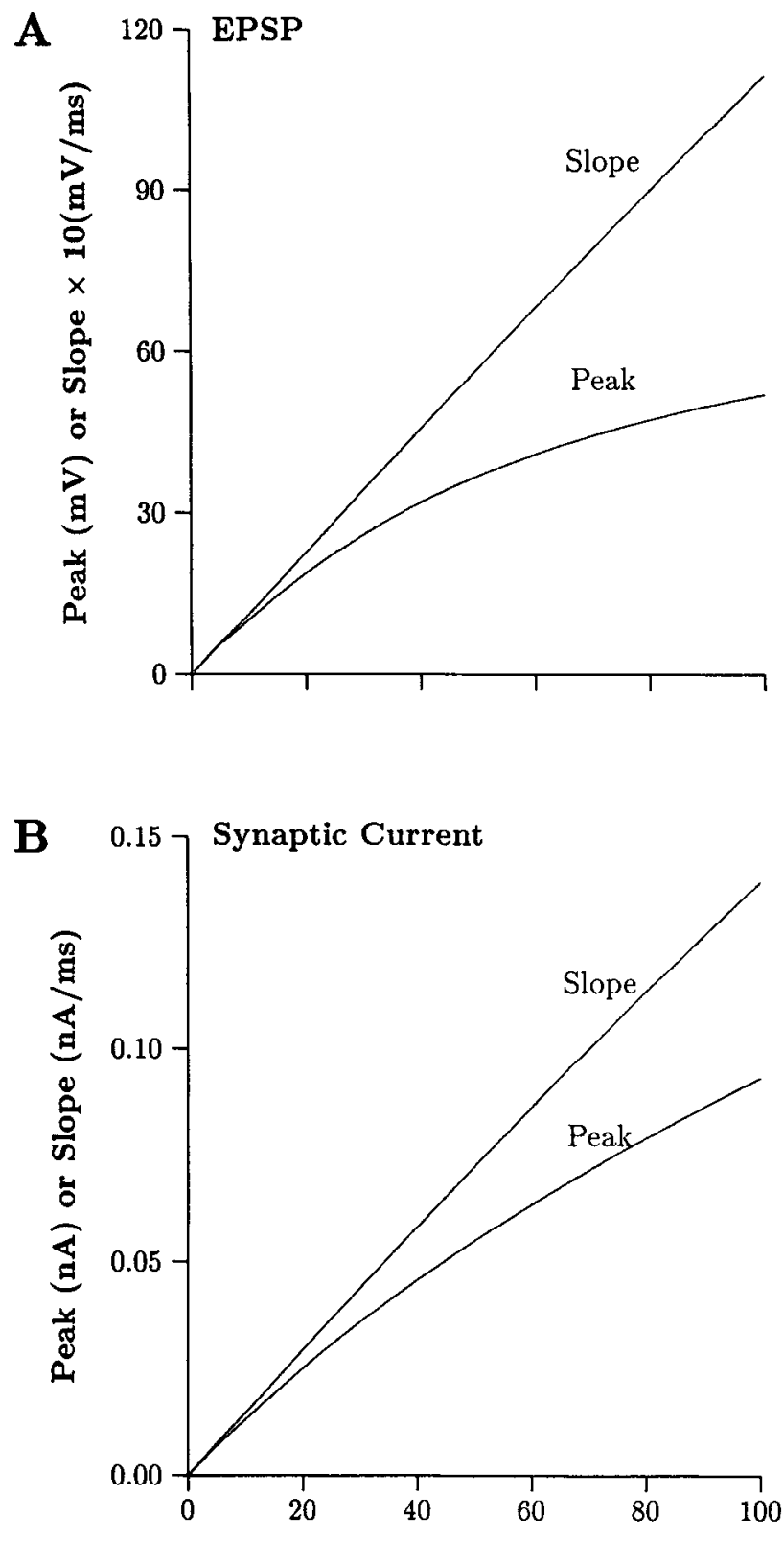

Total Peak Synaptic Conductance (nS)

Figure 8. Nonlinear summation can be expected of peak amplitudes but not slopes of synaptic currents and potentials. Simulations were performed to examine the effects on a single synapse of varying the synaptic conductance of 50 synapses placed in the apical dendrites of a single pyramidal neuron (see Materials and Methods). As total peak synaptic conductance was increased from $0.5 \mathrm{nS}$ to $100 \mathrm{nS}$, the initial slopes of the EPSPs $(A)$ and synaptic currents $(B)$ at a single synapse varied linearly with synaptic conductance; in contrast, the peak amplitudes of both exhibited nonlinear summation. These simulations suggest that initial pEPSP slope, which was used to measure PPF in our experiments, is unlikely to be contaminated by nonlinear summation.

have decreased by a similar amount. While short-term potentiation (STP) has a similar initial magnitude to PPF, it remained constant as LTP was saturated (Schulz and Johnston, 1991), suggesting that the changes in PPF are not due to nonlinear summation.

The lack of evidence for nonlinear summation of the pEPSP slope was somewhat unexpected. Thus, we examined nonlinear summation further through neural modeling. The effect on a single synapse of increasing the synaptic conductance of 50 synapses spread over the dendritic tree of a single pyramidal neuron was modeled (see Materials and Methods for details). As total peak synaptic conductance was increased from 0.5 to $100 \mathrm{nS}$, the peak amplitude of EPSPs (Fig. 8A) and synaptic currents (Fig. $8 B$ ) at single synapses exhibited pronounced nonlinear summation; in contrast, the initial slopes of both were linearly related to synaptic conductance. Since the extracellularly measured pEPSP in the dendrites is a reasonable measure of the underlying synaptic currents (Hubbard et al., 1969), this model predicts that the early pEPSP slopes measured in the present experiments would not exhibit nonlincar summation. This result can be explained by considering that the initial slope is an estimate of the initial synaptic current that flows when the driving force is maximal and unaffected by the synaptic potential. Later peak synaptic current would be affected by both changes in potential and a decrease in driving force.

\section{Changes in inhibition were not responsible for changes in PPF}

A sixth test of the specificity of changes in PPF to LTP was performed by examining whether the observed changes in PPF were due to changes in GABA-mediated inhibition. All the experiments reported in this study that were performed in the moderate- or high-calcium solutions had $10 \mu \mathrm{M}$ picrotoxin in the bathing solution to decrease $\mathrm{GABA}_{\mathrm{A}}$-mediated inhibition. Since changes in PPF were nonetheless induced, and were similar to those induced without picrotoxin present (in Fig. 1 compare normal-calcium slices, which had no picrotoxin, to moderate- and high-calcium slices with picrotoxin), it suggests that changes in $\mathrm{GABA}_{\mathrm{A}}$-mediated inhibition were not responsible for them. To determine whether changes in $G_{A B A}$-mediated inhibition were responsible for the changes in PPF with LTP, additional experiments were performed with both picrotoxin and $200 \mu \mathrm{M}$ 2-hydroxysaclofen, a $\mathrm{GABA}_{\mathrm{B}}$ antagonist, in the bathing solution.

2-Hydroxysaclofen at $200 \mu \mathrm{M}$ was chosen because 10,40 , and $400 \mu \mathrm{M}$ had previously been shown to decrease $\mathrm{GABA}_{\mathrm{B}}$-mediated inhibition in the dentate gyrus by $83 \%, 93 \%$, and $100 \%$ (Mott et al., 1993). Its $\mathrm{GABA}_{\mathrm{B}}$ blocking effect in CA1 was confirmed by us as follows. Baclofen $(10 \mu \mathrm{M})$, a GABA ${ }_{\mathrm{B}}$ agonist, was washed in and resulted in a $56 \%$ depression of the pEPSP slope (Fig. 9A, $\mathrm{;} ; n=4$, high-calcium solution), which was reversed by wash-out. Wash-in of $200 \mu \mathrm{M}$ 2-hydroxysaclofen for $25 \mathrm{~min}$ in two of those slices before wash-in of $10 \mu \mathrm{M}$ baclofen with $200 \mu \mathrm{M} 2$-hydroxysaclofen resulted in only a $10 \%$ depression of the pEPSP slope (Fig. $9 A$. $\bullet ; n=2$ ). Thus, $200 \mu \mathrm{m}$ 2-hydroxysaclofen blocked at least $82 \%$ of the baclofen-induced $\mathrm{GABA}_{\mathrm{B}}$-mediated inhibition of the pEPSP. Saclofen itself may have depressed pEPSPs (see Fig. $9 B$ ), so the estimate of an $82 \%$ blockade of $\mathrm{GABA}_{\mathrm{B}}$ receptors may be low. As an additional test of the $\mathrm{GABA}_{\mathrm{B}}$-blocking effect of $200 \mu \mathrm{M}$ 2-hydroxysaclofen, its effect on paired-pulse depression (PPD) was tested. When measured in four slices with ISIs of 200,300, and $400 \mathrm{msec}$, PPD averaged $13.2 \pm 4.8 \%$ (data not shown). When measured in two slices in the presence of saclofen with the same ISIs, average PPD was reduced to $2.3 \pm 6.5 \%$.

We then tested whether PPF changed in association with LTP when GABA-mediated inhibition was reduced. Picrotoxin at $10 \mu \mathrm{M}$ was already in the bath. Wash-in of 2-hydroxysaclofen was associated with a decrease in the evoked pEPSPs from 0.94 to $0.80 \mathrm{mV} / \mathrm{msec}$ (Fig. $9 B ; n=5$, high-calcium solution, initial 
pEPSP magnitude $=1.3 \mathrm{mV}$ ), and a small, nonsignificant increase in PPF from $22.6 \pm 3.4 \%$ to $25.8 \pm 2.4 \%$ (Fig. $9 C ; n=$ $5, p=0.53$, ISI $=55 \mathrm{msec}$ ). HFS (arrow) was associated with $18 \%$ LTP (Fig. $9 B$ ) and an increase in PPF from $25.8 \pm 2.4 \%$ to $34.4 \pm 1.5 \%$ (Fig. $9 C ; n=5$ ). The increase in PPF was significant by $t$ tests when compared either to baseline ( $p=$ $0.025)$ or after saclofen wash-in $(p=0.024)$. Since significant changes in PPF occurred in association with LTP despite decreased $\mathrm{GABA}_{\mathrm{A}}$ - and $\mathrm{GABA}_{\mathrm{B}}$-mediated inhibition, it suggests that activation of these receptors was not necessary for, and did not underlie, the changes we observed in PPF associated with LTP.

\section{PPF changed over a range of interstimulus intervals}

A seventh test of the specificity of the changes in PPF to LTP was performed by examining whether PPF changed over a broad range of ISIs in association with LTP. A presynaptic change associated with LTP would be expected to modify the PPF elicited by all ISIs, whereas PPF altered by another process might affect only certain ISIs. The PPF values plotted in previous figures were all obtained with a $55 \mathrm{msec}$ ISI, so we now examined the changes in PPF associated with LTP when PPF was elicited over a broader range of ISIs.

PPF was initially examined at baseline over ISIs of 15-400 msec. The greatest initial PPF was elicited with ISIs of 55-95 msec, though PPF was observed over the entire range. With ISIs of 15-45 msec, PPF varied with stimulus intensity (data not shown). Since pEPSP duration was from 15 to over $30 \mathrm{msec}$, it is likely that PPF measured with those short ISIs ( $\leq 45 \mathrm{msec}$ ) was affected by residual synaptic potentials. Paired-pulse depression was sometimes observed at ISIs of $200-400 \mathrm{msec}$ (data not shown). Because of possible interference by these other phenomena, then, PPF measured with ISIs of $45 \mathrm{msec}$ or less, and $200 \mathrm{msec}$ or greater were not used for examining changes in PPF associated with LTP.

The effect of LTP on PPF measured over a range of ISIs was then examined by eliciting PPF over ISIs of 55-150 msec at baseline, saturating LTP, and then remeasuring PPF over the same ISIs. Initial pEPSPs were all $1.3 \mathrm{mV}$. In moderate-calcium, an average of $93 \%$ LTP was elicited and was associated with a decrease in PPF, averaged over all ISIs, from $23.8 \pm 1.4 \%$ to $13.6 \pm 1.2 \%$ (Fig. $10 A ; n=7$, includes all slices in which PPF decreased and in which the range of ISIs was examined). In high-calcium, LTP saturation increased the initial pEPSP slope by $159 \%$ over baseline and was also associated with a decrease in PPF averaged over all ISIs from $15.9 \pm 2.0 \%$ to $7.4 \pm 1.6 \%$ (Fig. $10 B ; n=4$, includes all slices in which PPF decreased and in which the range of ISIs was examined). We conclude that the changes in PPF associated with LTP were not specific to one ISI, but occurred over a broad range of ISIs.

\section{Increased presynaptic calcium decreases PPF}

At least seven sets of experiments, then, suggested that the changes we observed in PPF were specific to LTP. This, in turn, suggested that the locus of LTP expression includes the presynaptic site. We wondered what type of presynaptic mechanism underlying LTP could result in both increases and decreases in PPF. One possible mechanism underlying LTP would be an increase in presynaptic calcium influx that would increase the probability of transmitter release. We reexamined the effect on PPF of increasing presynaptic calcium by two maneuvers.

First, the effect of increasing bath calcium was examined.
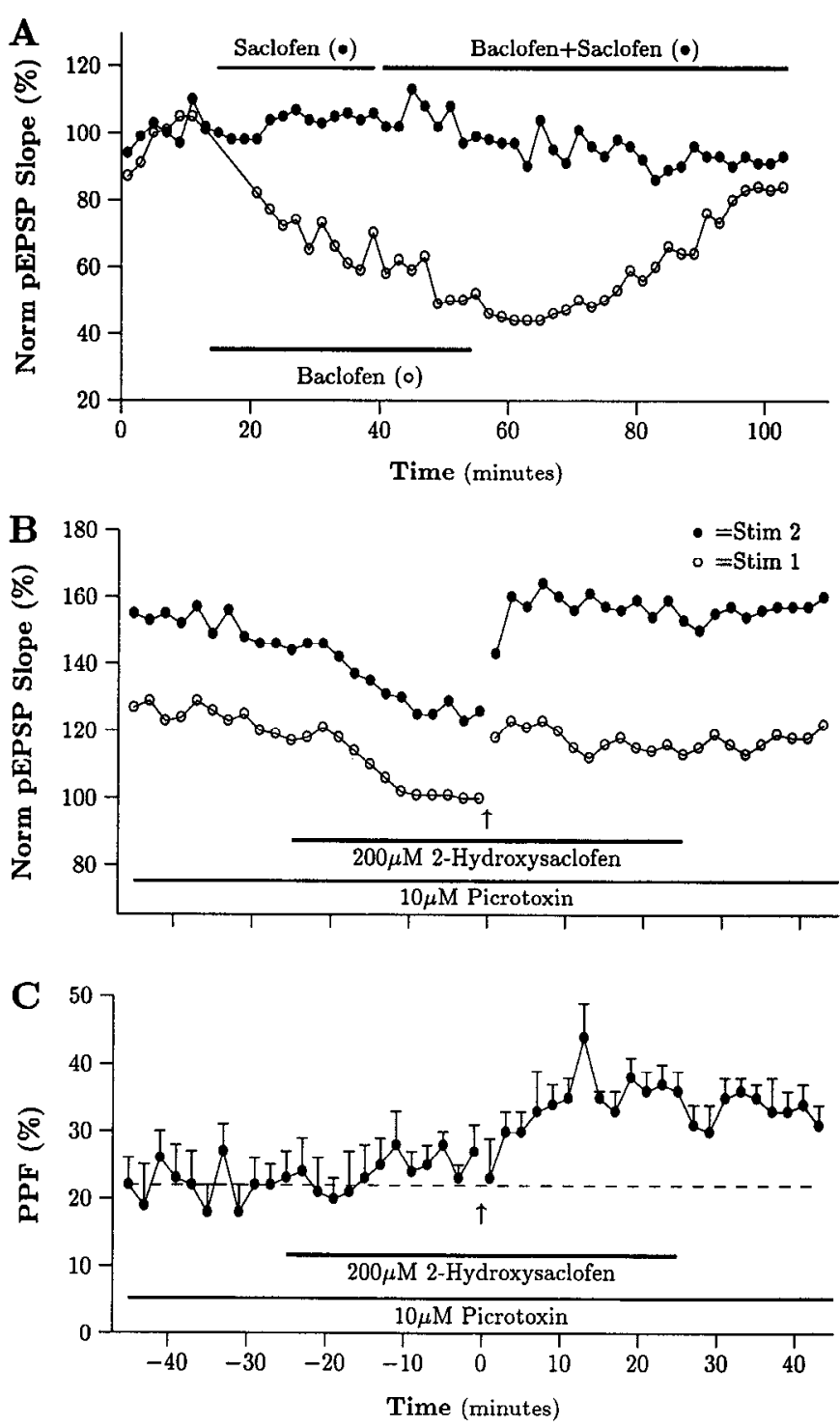

Figure 9. Changes in GABA-mediated inhibition do not underlie the changes in PPF associated with LTP. $A$, Baclofen $(10 \mu \mathrm{M})$, a GABA agonist, depressed the pEPSP slope by $56 \%(O ; n=4)$ and the effect was reversed by wash-out. Wash-in of $200 \mu \mathrm{M}$ 2-hydroxysaclofen, a $\mathrm{GABA}_{\mathrm{B}}$ antagonist, before $10 \mu \mathrm{M}$ baclofen with 2-hydroxysaclofen resulted in only a $10 \%$ decrease in slope so that saclofen blocked at least $82 \%$ of the baclofen-induced depression $(0 ; n=2)$. Some of that remaining $10 \%$ depression may have been saclofen induced as suggested by $B . B$ and $C$, In the presence of $10 \mu \mathrm{M}$ picrotoxin (a GABA antagonist), wash-in of $200 \mu \mathrm{M}$ 2-hydroxysaclofen depressed the pEPSP slope by $17.5 \%(B ; n=5$, high-calcium solution, initial pEPSP magnitude $=1.3$ $\mathrm{mV}$ ) and was associated with a small, nonsignificant increase in PPF from $22.6 \pm 3.4 \%$ to $25.8 \pm 2.4 \%(C$; ISI $=55 \mathrm{msec}, p=0.53$ by $t$ test). A subsequent single HFS (arrow) resulted in $18 \%$ LTP $(B)$ and was associated with a significant increase in PPF from $25.8 \pm 2.4 \%$ to $34.4 \pm 1.5 \%(C$; by $t$ tests, $p=0.024$ vs after saclofen wash-in and $p$ $=0.025$ vs baseline). These results suggest that the changes in PPF associated with LTP are not due to changes in GABA-mediated inhibition since the PPF changes were observed despite the reduction of GABA-mediated inhibition.

After obtaining stable $0.5 \mathrm{mV}$ pEPSPs, the extracellular calcium was increased by switching from the high-magnesium to the high-calcium bathing solution. This was associated with a $740 \%$ increase in pEPSP slope (Fig. $11 A, 0 ; n=3$ ), and a decrease in PPF from $50.5 \%$ to $16 \%$ (Fig. $11 B$ ), in agreement with previous reports showing that PPF decreases with increased presynaptic 

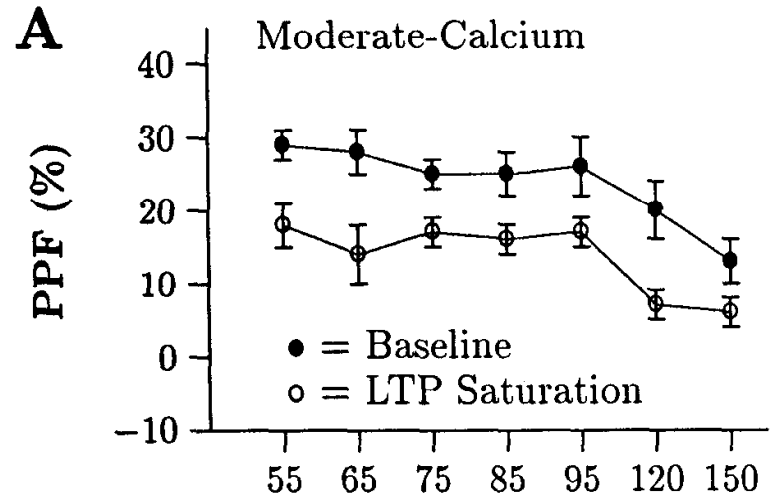
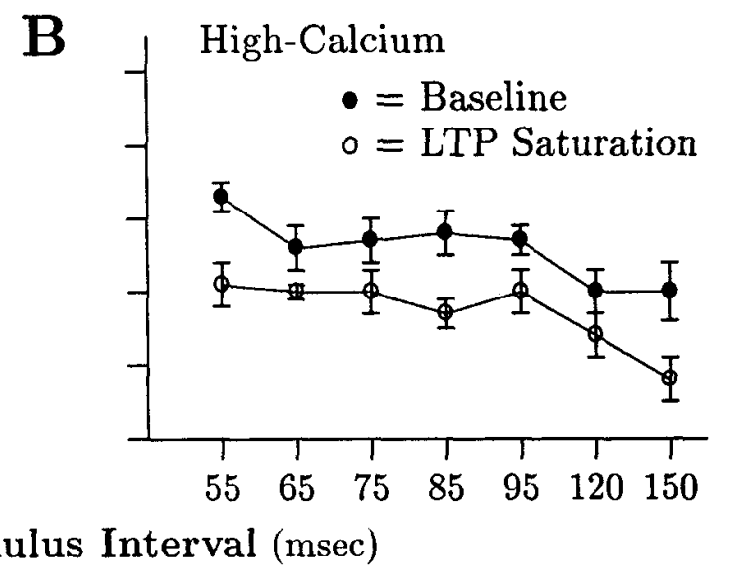

Figure 10. Decreases in PPF associated with LTP occurred over a broad range of ISIs. PPF was measured at baseline and after LTP saturation, over ISIs of $55-150 \mathrm{msec}$, in moderate- $(A)$ and high-calcium $(B)$. In saturating LTP, the average initial pEPSP slopes increased by $93 \%(A ; n=$ 7) and $159 \%(B ; n=4)$. PPF decreased over the entire range of ISIs in both solutions as expected from a presynaptic process. Note that the $\mathrm{x}$-axis is not linear.

calcium infiux (Asztely et al., 1990). While the extracellular calcium concentration was increasing, PPF did not increase in any of the slices. Superimposition of the first and second pairedpulse pEPSPs elicited with an ISI of $55 \mathrm{msec}$ in Figure $11 \mathrm{C}$ demonstrates that PPF measured in lower calcium was greater (a) than in the higher calcium solution (b).

Second, the effect of PTP on PPF was examined. The first HFS in Figure $6 A$, in which HFS was given in $100 \mu \mathrm{M}$ D,L-APV, resulted in short-lived PTP of $28 \%(n=3)$. Figure $6 B$ demonstrates that this PTP was associated with a decrease in PPF from $27.6 \pm 1.4 \%$ to $-2.2 \pm 4.2 \%$ (ISI $=55 \mathrm{msec}$ ), in agreement with previous studies (Anwyl et al., 1989).

In agreement with previous studies, then, conditions associated with an increase in presynaptic calcium were associated with larger pEPSPs, presumably due to an increased probability of transmitter release, and were associated only with decreases in PPF, not increases. Since we observed both increases and decreases in PPF associated with LTP, a simple increase in presynaptic calcium influx would not appear to underlie LTP expression. Other investigators have performed optical measurcments of presynaptic calcium in association with LTP and have also found no evidence for changes in calcium influx ( $\mathrm{Re}$ gehr and Tank, 1991; Wu and Saggau, 1994). The hypothesis that LTP is mediated by an increase in presynaptic calcium would also predict that less LTP would be induced in highcalcium than in low-calcium because the increased extracellular calcium would already have increased release towards its limit. The same amount of LTP was induced, however, in all three solutions.

\section{Discussion}

This study found that PPF changed in association with LTP and the change was inversely related to initial PPF magnitude so that a larger initial PPF was associated with a decrease in PPF while a smaller initial PPF was associated with an increase. While PPF in individual slices changed with LTP, average PPF remained constant. This study also demonstrated that the changes in PPF were specific to LTP as similar amounts of LTP were associated with similar changes in PPF, PPF changed with the same time course as LTP, and changes in PPF were not due to nonspecific effects of HFS, were input specific, were not due to active postsynaptic currents or their nonlinear summation, did not involve changes in inhibition, and occurred over a range of interstimulus intervals.

Previous studies found no change in average PPF associated with LTP. Our original hypothesis as to why other studies might not have reported a change in PPF with LTP, if it existed, was that insufficient LTP had been induced. To test this hypothesis we initially maximized LTP by using multiple HFS, high-calcium saline, and GABA antagonists. While we noted changes in PPF under these conditions (Schulz and Johnston, 1992), we subsequently found that changes in PPF occurred even with the modest amounts of LTP induced by single HFS (Fig. $2 B$ ), in normal-calcium (Fig. $1 B$ ), and with intact inhibition (Fig. 1B). Thus, our original hypothesis as to why changes in PPF had not previously been observed was incorrect. Finding that PPF increased and decreased with LTP, and that average PPF therefore remained constant, however, suggests the more probable reason why changes in PPF were not previously observed. Previous hypotheses had suggested that PPF would only decrease in association with LTP, if it changed at all. Thus, it was reasonable in previous studies to compare average PPF at baseline to average PPF after LTP induction. If PPF had both increased and decreased in association with LTP, however, it might not have been recognized because average PPF would have remained constant.

Experiments using alternate techniques have also suggested that there is presynaptic involvement in LTP; however, there has been a conflict in many instances between such results and opposite results obtained by other groups using the same techniques (see introductory remarks). In addition, intcrpreting the results of experiments using several techniques are difficult because they involve assumptions that have not been verified. Thus, the simplicity of the technique used here (examining PPF), the paucity of assumptions involved (that only a presynaptic process is being measured), and the apparent validity of that assumption suggest that this may be some of the strongest evidence yet for presynaptic involvement in LTP.

\section{Changes in PPF were specific to LTP}

Finding that PPF changed in association with LTP supports the hypothesis that LTP expression includes the presynaptic locus 
since previous studies have shown that a presynaptic mechanism underlies PPF in CA1 (Foster and McNaughton, 1991; Storm, 1992). The fact that PPF increased, decreased, or remained the same with LTP, however, made it especially important to demonstrate that the changes in PPF were specific to LTP. Thus, we addressed a number of alternate mechanisms by which PPF might change in association with HFS.

First, several lines of evidence suggest that the changes in PPF were not due to the nonlinear summation of postsynaptic currents. (1) Nonlinear summation predicts only decreases in PPF and would not account for the increases observed. (2) Returning the pEPSPs to baseline with CNQX after LTP induction did not return PPF to baseline values (Figs. 3, 4). (3) STP did not show decreases in amplitude despite being of similar amplitude to PPF (Schulz and Johnston, 1991). (4) Neural modeling suggested that over the range of synaptic currents likely to be encountered in the hippocampus, nonlinear summation of the pEPSP slope was unlikely to occur (Fig. 8). (5) Activation of an excitatory postsynaptic current would be unlikely to both incrcasc and dccrcase PPF. (6) Changes in inhibitory currents also do not appear to have interfered with our measure of PPF, as discussed below.

Second, several lines of evidence suggest that the changes in PPF cannot be attributed to changes in inhibition. (1) PPF changes were observed in the presence of inhibitors of both $\mathrm{GABA}_{\mathrm{A}}$ and $\mathrm{GABA}_{\mathrm{B}}$ receptors (Fig. 9). While one could argue that neither picrotoxin nor 2-hydroxysaclofen completely blocked $\mathrm{GABA}_{A}$ and $\mathrm{GABA}_{\mathrm{B}}$ receptors, the fact that similar changes in PPF occurred in their presence or absence suggests that GABAmediated inhibition was not responsible for changes in PPF. (2) A single change in inhibition would not appear to account for both increases and decreases in PPF. (3) The various forms of inhibition have limited time courses over which they produce effects. PPF, however, changed over a range of interstimulus intervals (Fig. 10). (4) Short-lasting changes in inhibitory postsynaptic potentials in CAl have been reported to occur during (Davies et al., 1991) or after HFS (McCarren and Alger, 1985), but generally do not persist beyond a minute or two. (5) If a change in the balance of excitation to inhibition were responsible for the changes observed in PPF, then decreasing the excitatory PSP with CNQX might have altered PPF by also altering the balance of excitation to inhibition, but PPF did not change with CNQX (Figs. 3, 4).

Third, the changes in PPF were not due to a nonspecific effect of HFS as APV blocked both LTP and changes in PPF (Fig. 6). In addition, after LTP saturation, HFS did not produce additional LTP or additional changes in PPF. Fourth, slices that had a change in fiber volley or stimulus artifact were not included so that nonspecific short-term changes in excitability and slice movement would not appear to account for changes in PPF. Fifth, the changes in PPF associated with LTP were specific to the input exhibiting LTP (Fig. 7) so that a gencralized change in PPF in the slice, as well as a generalized change in postsynaptic excitability, did not appear to occur. Sixth, a decline in slice health due, for example, to advanced slice age would not appear to account for the changes in PPF since they were rapid (see Fig. 4) and stable (see Figs. 3, 4).

Finally, several changes in postsynaptic receptor numbers or properties might be hypothesized to have effected PPF. In general, the addition of postsynaptic receptors or changes in their conductivity with LTP should not alter PPF, which is measured as a percentage change. In addition, the lack of change in STP
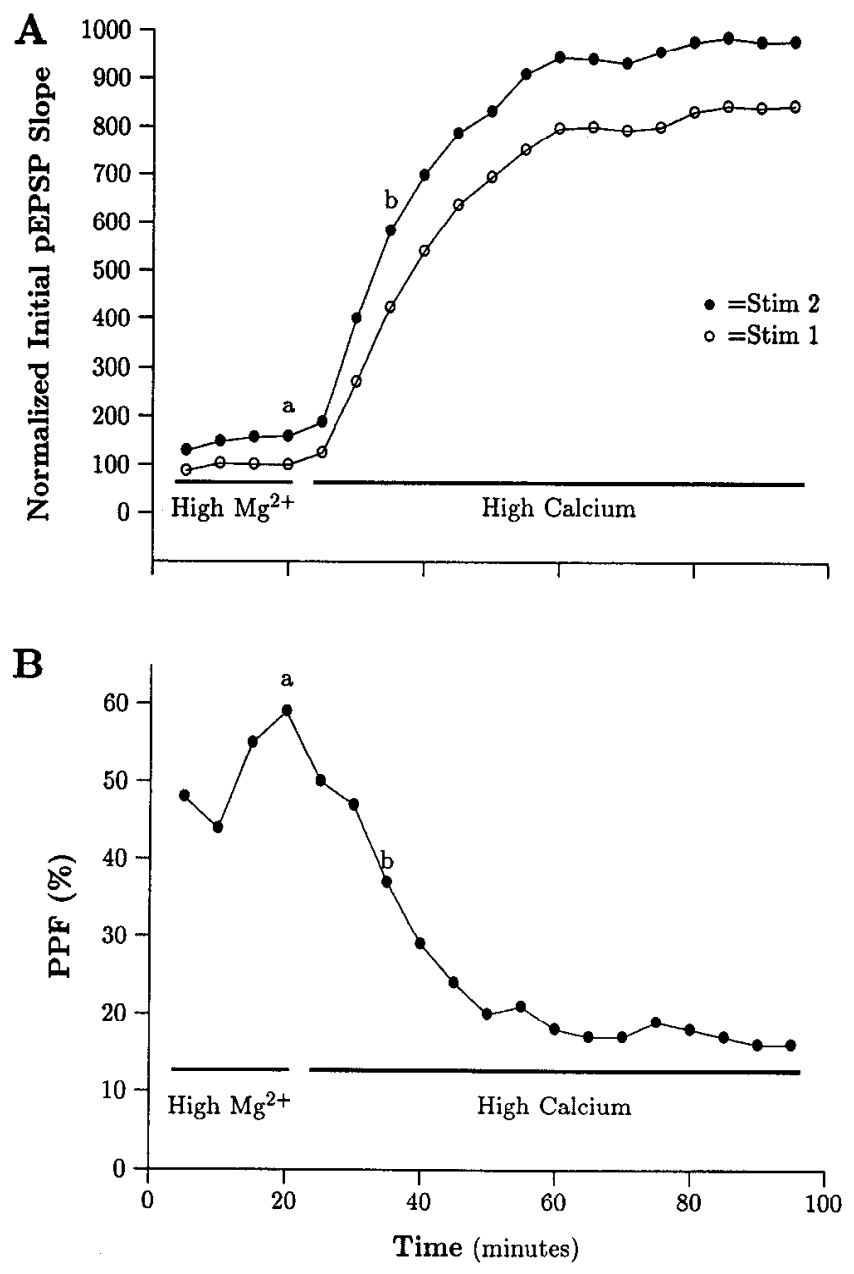

C
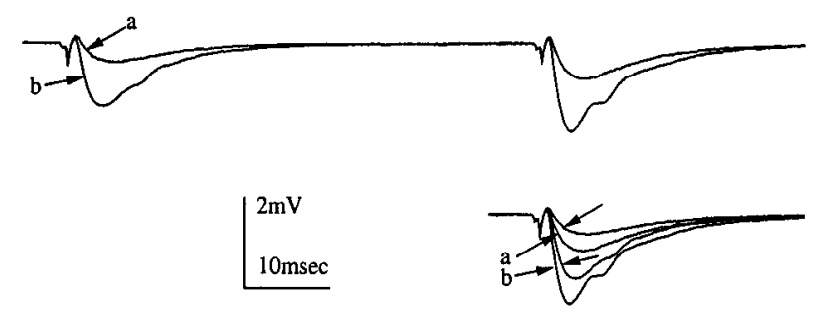

Figure 11. Increased extracellular calcium is associated with a decrease in PPF. $A$, The normalized pEPSP slopes of the first and second pairedpulse stimuli measured with an ISI of $55 \mathrm{msec}$ are plottcd. Aftcr obtaining a stable baseline in high-magnesium, the perfusing solution was changed to the high-calcium one. The change of solution was associated with a $740 \%$ increase in pEPSP slope (O; baseline $=100 \%, n=3) . B$, The increase in pEPSP slope was associated with a decrease in PPF from $50.5 \%$ to $16 \%$, indicating that increased presynaptic calcium is associated with a decrease in PPF. Each point is the average of $5 \mathrm{~min}$ of data, collected at $0.05 \mathrm{~Hz}$, and averaged over all experiments. $C$, Superimposition of raw voltage traces, each of which is the average of several traces, obtained at times $a$ and $b$, indicates that PPF in lower calcium $(a)$ is larger than in higher calcium $(b)$.

with LTP induction (Schulz and Johnston, 1991) may also argue against the addition of new receptors or an increase in their conductivity. A second concern might be that postsynaptic receptor activity would be saturated by LTP induction, and its saturation might decrease PPF. It would be difficult to understand, then, how PPF would increase in other slices, and how other forms of potentiation would still be expressible, for ex- 
ample, PPF, PTP, and STP. Moreover, saturation of receptors during LTP is also most easily explained by a presynaptic mechanism of LTP. A third concern would be that LTP might involve the addition of postsynaptic receptors so that PPF might increase by "unsaturating" the postsynaptic response. That hypothesis would not account, however, for the decreases in PPF also observed with LTP.

Thus, multiple experiments argue that the changes observed in PPF are specific to LTP. But what type of presynaptic mechanism might underlie LTP such that it would be associated with both increases and decreases in PPF?

\section{The presynaptic change associated with LTP}

In attempting to understand the possible presynaptic mechanisms for LTP that might account for our results, we started with the assumptions (1) that transmitter release is proportional to the product of the number of neurotransmitter release sites $(n)$ and the average probability of neurotransmitter release $(p)$ at those sites, or release $=n p$; and (2) that PPF reflects only changes in $p$. Using these assumptions, if LTP were associated only with an increase in $p$, then PPF would be predicted to only decrease with LTP. We, however, observed both increases and decreases in PPF with LTP. If LTP were associated with an increase in $n$ exclusively, and those release sites had the same average $p$ as the original release sites, then there would be no predicted interaction between LTP and PPF. Again, we observed changes in PPF with LTP. On the other hand, if the new release sites had both greater and lesser probabilities of release than the original release sites, then the measured PPF could both decrease and increase with LTP.

Another presynaptic mechanism that might account for our results is that LTP could be associated with increases in both $p$ and $n$, but with the new release sites having a low probability of release. This explanation would proceed as follows: PPF could be decreascd by incrcases in $p$, and increased by the addition of release sites with a low $p$. Measured PPF would decrease or increase with LTP depending on whether LTP were due predominantly to an increase in $p$ or $n$. In the slices in which initial $p$ was low (PPF high, right side of Fig. $1 A$ ), LTP might be mediated by an increase in $p$ and the resulting measured PPF would decrease. In contrast, when initial $p$ was high (PPF low, left side of Fig. $1 A$ ) so that there was little range for increase, LTP would be predominantly mediated by an increase in $n$. In this case the measured PPF would incrcasc. The number of release sites might increase under both conditions, but its effect would be more obvious when there is little change in $p$.

The notion that new release sites might have different $p$ values than the baseline sites is not unreasonable. There appears to be considerable diversity of $p$ values in slices; for example, at baseline in a single solution, we observed initial PPF values from $10 \%$ to $72 \%$ (normal-calcium; Fig. $1 B$ ). Other work has also suggested that there are differing probabilities of release at baseline (Hessler et al., 1993; Rosenmund et al., 1993). Thus, it seems possible that new release sites could have a different average $p$ than the average $p$ of the originally sampled population.

The possibility of two processes contributing to LTP expression was raised in two previous reports where it was suggested that when initial $p$ is low, the predominant change responsible for LTP is presynaptic, whereas when initial $p$ is high, the predominant change is postsynaptic (Larkman et al., 1992; Liao et al., 1992). Because PPF does not measure postsynaptic changes, our experiments suggest that when initial $p$ is high, there may also be a change in $n$ associated with LTP.

This study has demonstrated that PPF changes predictably in association with LTP. The changes in PPF appear to be specific to LTP as demonstrated by multiple control experiments. The specific changes in PPF, in turn, support the hypothesis that the mechanism of LTP expression includes the presynaptic site. An increase in $p$ and $n$, or possibly $n$ alone, appears to be the best explanation for the changes observed in PPF with LTP. The findings presented here do not rule out a postsynaptic component to LTP expression, and further work is clearly needed to correlate specific pre- and postsynaptic processes to LTP.

\section{References}

Andersen P, Sundberg SH, Sveen O, Swann JW, Wigstrom H (1980) Possible mechanisms for long-lasting potentiation of synaptic transmission in hippocampal slices from guinea pigs. J Physiol (Lond) 302 463-482.

Aniksztejn L, Roisin MP, Amsellem R, Ben-Ari Y (1989) Long-term potentiation in the hippocampus of the anaesthetized rat is not associated with a sustained enhanced release of endogenous excitatory amino acids. Neuroscience 28:387-392.

Anwyl R, Mulkeen D, Rowan MJ (1989) The role of $N$-methyl-Daspartate receptors in the generation of short-term potentiation in the rat hippocampus. Brain Res 503:148-151.

Asztely F, Hanse E, Gustafsson B (1990) The early decay of long-term potentiation in the hippocampal CAl region in vitro is reduced by activators of protein kinase C. Brain Res 521:355-358.

Augustine GJ, Charlion MP, Smith SJ (1987) Calcium action in synaptic transmitter release. Annu Rev Neurosci 10:633-693.

Bashir ZI, Alford S, Davies SN, Randall AD, Collingridge GL (1991) Long-term potentiation of NMDA receptor-mediated synaptic transmission in the hippocampus. Nature 349:156-158.

Baudry M, Oliver M, Creager R, Wieraszko A, Lynch G (1980) Increase in glutamate receptors following repetitive electrical stimulation in hippocampal slices. Life Sci 27:325-330.

Bekkers JM, Stevens CF (1990) Presynaptic mechanism for long-term potentiation in the hippocampus. Nature 346:724-728.

Brown TH, Chapman PF, Kairiss EW, Keenan CL (1988) Long-term synaptic potentiation. Science 242:724-728.

Christie BR, Abraham WC (1994) Differential regulation of pairedpulse plasticity following LTP in the dentate gyrus. Neuroreport 5 385-388.

Davies CH, Starkey SJ, Pozza MF, Collingridge GL (1991) GABA autoreceptors regulate the induction of I.TP. Nature 349:609-611.

Davies SN, Lester RAJ, Reymann KG, Collingridge GL (1989) Temporally distinct pre- and post-synaptic mechanisms maintain longtcrm potentiation. Nature 338:500-503.

Delaney KR, Zucker RS, Tank DW (1989) Calcium in motor nerve terminals associated with posttetanic potentiation. J Neurosci 9:35583567.

Dolphin AC, Errington ML, Bliss TVP (1982) Long-term potentiation of the perforant path in vivo is associated with increased glutamate release. Nature 297:496-498.

Foster TC, McNaughton BL (1991) Long-term synaptic enhancement in CAl is due to increased quantal size, not quantal content. Hippocampus 1:79-91.

Ghijsen WEJM, Da Silva FHL (1991) Increase in endogenous amino acid release in long-term potentiation. In: Long-term potentiation: a debate of current issues (Baudry M, Davis JL, eds), pp 45-55. Cambridge: MIT Press.

Goh JW, Pennefather PS (1989) A pertussis toxin-sensitive $G$ protein in hippocampal long-term potentiation. Science 244:980-982.

Haas HL, Schaerer B, Vosmansky M (1979) A simple perfusion chamber for the study of nervous tissue slices in vitro. J Neurosci Methods 1:323-325.

Harris KM, Jensen FE, Tsao BH (1989) Ultrastructure, development, and plasticity of dendritic spine synapses of area CAl of the rat hippocampus: extending our vision with serial electron microscopy and three-dimensional analyses. In: Neurology and neurobiology se- 
ries, Vol 52, The hippocampus: new vistas (Chan-Palay V, Köhler C, eds), pp 33-52. New York: Liss.

Hessler NA, Shirke AM, Malinow R (1993) The probability of transmitter release at a mammalian central synapse. Nature 366:569-572.

Hines M (1989) A program for simulation of nerve equations with branching geometries. Int J Biomed Comput 24:55-68.

Hubbard JI, Llinás R, Quastel DMJ (1969) Extracellular field potentials in the central nervous system. In: Electrophysiological analysis of synaptic transmission, pp 265-293. London: Arnold.

Kauer JA, Malenka RC, Nicoll RA (1988) A persistent postsynaptic modification mediates long-term potentiation in the hippocampus. Neuron 1:911-917.

Krug M, Wagner M, Broedemann R, Matthies H (1989) Habituation of monosynaptic field potentials in the dentate gyrus of freely moving rats interferes with LTP. Brain Res 476:163-166.

Kullmann DM, Nicoll RA (1992) Long-term potentiation is associated with increases in quantal content and quantal amplitude. Nature 357: 240-244.

Larkman A, Hannay T, Statford K, Jack J (1992) Presynaptic release probability influences the locus of long-term potentiation. Nature 360 : 70-73.

Liao D, Jones A, Malinow R (1992) Direct measurement of quantal changes underlying long-term potentiation in CA1 hippocampus. Neuron 9:1089-1097.

Madison DV, Malenka RC, Nicoll RA (1991) Mechanisms underlying long-term potentiation of synaptic transmission. Annu Rev Neurosci 14:379-398.

Malgaroli A, Tsien RW (1992) Glutamate-induced long-term potentiation of the frequency of miniature synaptic currents in cultured hippocampal neurons. Nature 357:134-139.

Malinow R, Tsien RW (1990) Presynaptic enhancement shown by whole-cell recordings of long-term potentiation in hippocampal slices. Nature 346:177.

Malinow R, Schulman H, Tsien RW (1989) Inhibition of postsynaptic PKC and CaMKII blocks induction but not expression of LTP. Science 245:862-866.

Manabe T, Renner P, Nicoll RA (1992) Postsynaptic contribution to long-term potentiation revealed by the analysis of miniature synaptic currents. Nature 355:50-55.

Manabe T, Wyllie DJA, Perkel DJ, Nicoll RA (1993) Modulation of synaptic transmission and long term potentiation: effects on paired pulse facilitation and EPSC variance in the CA1 region of the hippocampus. J Neurophysiol 70:1451-1459.

McCarren M, Alger BE (1985) Use-dependent depression of IPSPs in rat hippocampal pyramidal cells in vitro. J Neurophysiol 53:557-571.

McNaughton BL (1982) Long-term synaptic enhancement and shortterm potentiation in rat fascia dentata act through different mechanisms. J Physiol (Lond) 324:249-262.

Mott DD, Xie C-W, Wilson WA, Swarrtzwelder HS, Lewis DV (1993) $\mathrm{GABA}_{\mathrm{B}}$ autoreceptors mediate activity-dependent disinhibition and enhance signal transmission in the dentate gyrus. J Neurophysiol 69: 674-691.

Mulkey RM, Malenka RC (1992) Mechanisms underlying induction of homosynaptic long-term depression in area $\mathrm{CAl}$ of the hippocampus. Ncuron 9:967-975.

Muller D, Lynch G (1988) Long-term potentiation differentially affects two components of synaptic responses in hippocampus. Proc Natl Acad Sci USA 85:9346-9350.
Muller D, Lynch G (1989) Evidence that changes in presynaptic calcium currents are not responsible for long-term potentiation in hippocampus. Brain Res 479:290-299.

Nelson RB, Linden DJ, Routtenberg A (1989) Phosphoproteins localize to presynaptic terminals linked to persistence of long-term potentiation (LTP): quantitative analysis of two-dimensional gels. Brain Res 497:30-42.

Regehr WR, Tank DW (1991) The maintenance of LTP at hippocampal mossy fiber synapses is independent of sustained presynaptic calcium. Neuron 7:451-459.

Rosenmund C, Clements JD, Westbrook GL (1993) Nonuniform probability of glutamate release at a hippocampal synapse. Science 262:754-757.

Routtenberg A, Lovinger D, Steward O (1985) Selective increase in the phosphorylation of a $47 \mathrm{kD}$ protein $(\mathrm{F} 1)$ directly related to longterm potentiation. Behav Neural Biol 43:3-11.

Schulz PE, Johnston D (1991) Evidence for differing mechanisms of short- and long-term potentiation expression. Soc Neurosci Abstr 17: 386.

Schulz PE, Johnston D (1992) Changes in paired-pulse facilitation (PPF) suggest presynaptic involvement in the expression of LTP in hippocampal area CA1. Soc Neurosci Abstr 18:403.

Skrede KK, Malthe-Sorenssen D (1981) Increased resting and evoked release of transmitter following repetitive electrical tetanization in hippocampus: a biochemical correlate to long lasting synaptic potentiation. Brain Res 208:436-441.

Spruston N, Williams SH, Jaffe DB, Johnston D (1993) Voltage- and space-clamp errors associated with the measurement of electrotonically remote synaptic events. J Neurophysiol 70:781-802.

Staubli U, Larson J, Lynch G (1990) Mossy fiber potentiation and long-term potentiation involve different expression mechanisms. Synapse 5:333-335

Storm JF (1992) Transmission-dependent depression coexists with facilitation at hippocampal excitatory synapses. Soc Neurosci Abstr 18:1340.

Swandulla D, Hans M, Zipser K, Augustine GJ (1991) Role of residual calcium in synaptic depression and posttetanic potentiation: fast and slow calcium signaling in nerve terminals. Neuron 7:915-926.

Teyler TJ, DiScenna P (1984) Long-term potentiation as a candidate mnemonic device. Brain Res Rev 7:15-28.

Teyler TJ, DiScenna P (1987) Long-term potentiation. Annu Rev Neurosci 10:131-161.

Voronin LL, Kuhnt U (1990) Long-term potentiation affects facilitation ration of EPSPs recorded from CAl pyramidal cells in the guinea pig hippocampal slice. Neurosci Res Commun 6:149-155.

Williams SH, Johnston D (1990) Muscarinic depression of synaptic transmission at the hippocampal mossy fiber synapse. J Neurophysiol 64:1089-1097.

Wu LG, Saggau P (1994) Presynaptic calcium is increased during normal synaptic transmission and paired-pulse facilitation, but not long-term potentiation in area $\mathrm{CAl}$ of hippocampus. J Neurosci 14: 645-654.

Zalutsky RA, Nicoll RA (1990) Comparison of two forms of longterm potentiation in single hippocampal neurons. Science 248:16191624 . 